\title{
The miR-200b-3p/ERG/PTHrP Axis Mediates the Inhibitory Effect of Ethanol on the Differentiation of Rat Fetal Chondrocytes into Articular Cartilage
}

\section{Qubo Ni}

Wuhan University Zhongnan Hospital

Haitao Chen

Wuhan University Zhongnan Hospital

Bin Li

Wuhan University Zhongnan Hospital

Hangyuan He

Wuhan University Zhongnan Hospital

Huasong Shi

Wuhan University Zhongnan Hospital

Jiayong Zhu

Wuhan University Zhongnan Hospital

Hui Gao

Wuhan University Zhongnan Hospital

Hui Wang

Wuhan University School of Basic Medical Sciences

Liaobin Chen ( $\square$ lbchen@whu.edu.cn )

Wuhan University Zhongnan Hospital https://orcid.org/0000-0002-8778-1457

\section{Research Article}

Keywords: Prenatal ethanol exposure, Articular cartilage, ERG, PTHrP, miR-200b-3p

Posted Date: June 29th, 2021

DOl: https://doi.org/10.21203/rs.3.rs-635407/v1

License: (c) (i) This work is licensed under a Creative Commons Attribution 4.0 International License.

Read Full License 


\section{Abstract \\ Background}

This study aims to further explore cartilage development in prenatal ethanol exposure (PEE) offspring at different times to explore the specific time points and mechanism of ethanol-induced fetal cartilage dysplasia.

\section{Methods}

On gestational day (GD)14, GD17, and GD20, PEE fetal cartilage was evaluated by morphological analysis. RT-qPCR, immunohistochemistry, and immunofluorescence were used to detect the expression of cartilage marker genes and their regulatory factors. Bone marrow mesenchymal stem cells (BMSCs) were used to explore the effect of ethanol on the differentiation of chondrocytes. Additionally, we used inhibitors, overexpression plasmids and a luciferase reporter assay on GD17 chondrocytes to verify the mechanism.

\section{Findings:}

PEE significantly reduced cartilage matrix content and the expression of marker genes on GD17 and GD20 but had no effect on GD14. The inhibition of chondrogenic differentiation by PEE mainly occurred on GD14-17. Furthermore, the expression of miR-200b-3p was increased, while that of ERG and PTHrP was markedly reduced in PEE fetal cartilage. In vitro, ethanol (30-120 mM) inhibited the differentiation of BMSCs into chondrocytes in a concentration-dependent manner, accompanied by strong expression of miR-200b-3p and low expression of ERG and PTHrP. Moreover, PTHLH and ERG overexpressed, as well as a miR-200b-3p inhibitor reversed the inhibitory effect of ethanol on the differentiation of fetal chondrocytes. Furthermore, miR-200b-3p could target and negatively regulate ERG.

\section{Interpretation:}

PEE can significantly inhibit the development of articular cartilage, especially during articular cartilage formation. The mechanism is related to the decreased differentiation of fetal cartilage into articular cartilage mediated by the miR-200b-3p/ERG/PTHrP axis.

\section{Introduction}

Osteoarthritis $(\mathrm{OA})$ is an age-related disease characterized by the progressive loss of articular cartilage, concomitant sclerotic changes in the subchondral bone, and the formation of osteophytes(Lawrence et al. 2008). The etiology of OA is complex, and involves age-related, genetic, developmental, and biomechanical factors(Shen et al. 2019). Epidemiological data suggest that people with low birth weight 
are susceptible to OA in adulthood, and a fetal origin of OA has been speculated(Aigner and Richter 2012; Jordan et al. 2005; Sayer et al. 2003). In a series of studies our team proposed for the first time and confirmed that OA has fetal origin(Chen et al. 2019a; Li et al. 2020; Tie et al. 2016a; Tie et al. 2016b). Prenatal ethanol exposure (PEE) can lead to postnatal articular cartilage dysplasia and susceptibility to OA in adulthood(Ni et al. 2015a; Ni et al. 2018; Ni et al. 2015b); however, the specific details and mechanisms remain undefined. Articular cartilage plays an important role in OA. Chondrocytes are the only cell component of articular cartilage; they are mainly formed in the intrauterine period, and subtle changes in cartilage development may induce the occurrence of OA(Kornak and Mundlos 2003; Pitsillides and Beier 2011). The underlying reasons are related to the changes in the force line, geometric characteristics and cartilage matrix composition of articular cartilage caused by chondrodysplasia(Kornak and Mundlos 2003; Pitsillides and Beier 2011). These observations suggest that the development of articular cartilage in embryos is very important for the quality of adult articular cartilage.

Articular cartilage originates from mesodermal mesenchymal stem cells. At the 6th week of human embryo development, mesenchymal cells in the central axis of branch buds increase and aggregate to form the limb bud. At the initial stage, there is no joint space in the limb bud, and after 2 weeks of differentiation and development, the joints are formed(Bukowska et al. 2018; Shapiro 2001). The development process of the rat skeletal system is very similar to that of humans, so rats are still the main animal model for skeletal research(Abubakar et al. 2016; Blaker et al. 2017). In rats, by gestational day (GD)11, mesenchymal precursor cells have differentiated into prechondrocytes that have aggregated to form a cartilage anlage. Chondrocytes in the center of the cartilage anlage undergo hypertrophic differentiation and endochondral osteogenesis to form long bones that determine body length, which is called transitional cartilage. Chondrocytes at both ends of the cartilage anlage do not undergo hypertrophic differentiation and maintain their function throughout life, eventually forming articular cartilage, which is called permanent cartilage(Kronenberg 2003; Pacifici et al. 2005). This suggests that the transient chondrocytes involved in endochondral osteogenesis share the same tissue origin as the permanent chondrocytes that form articular cartilage. Ray and his colleagues also confirmed this viewpoint using lineage tracing and pulse-chase methods(Ray et al. 2015). ETS-related gene (ERG) belongs to the ETS family, which regulates the differentiation, development, and permanent-like status of cartilage progenitor cells (Iwamoto et al. 2005; Iwamoto et al. 2001; Iwamoto et al. 2007). Studies have confirmed that ERG can promote the expression of tenascin- $\mathrm{C}$ in articular chondrocytes and inhibit the expression of col10a1 in hypertrophic chondrocytes(Iwamoto et al. 2005; Ohta et al. 2015). Parathyroid hormone-related protein (PTHrP encoded by PTHLH) is a key regulator of transitional chondrocytes and is secreted by articular chondrocytes and perichondrium cells. PTHrP was reported to maintain the continuous proliferation of chondrocytes and inhibit their terminal differentiation into hypertrophic chondrocytes after mitosis(Pelosi et al. 2013; Singh et al. 2018). One study has also shown that the partial regulatory effect of ERG on chondrocytes is mediated by PTHrP(Okabe et al. 2011). This finding shows that ERG can regulate the differentiation and developmental direction of embryonic cartilage progenitor cells through PTHrP. 
MicroRNAs (miRNAs) are small single-stranded noncoding RNAs that play pivotal roles by regulating functional genes in the process of growth and development(Martinez and Peplow 2021; Razmara et al. 2019). Multiple studies have demonstrated that miRNAs play a crucial role in regulating stem cell differentiation and maintaining stem cell characteristics(Barter et al. 2015; Miyaki et al. 2010; Swingler et al. 2012). Currently, the role of miRNAs in the development of articular cartilage has been confirmed by an increasing number of studies, and many miRNAs have been verified to participate in the proliferation, hypertrophy, differentiation and development of chondrocytes(Chen et al. 2019b; Le et al. 2020; Suomi et al. 2008). A large number of studies have also confirmed that miRNAs play a key regulatory role in cartilage development and adult related diseases(Coutinho de Almeida et al. 2019; Kang et al. 2019; Kobayashi et al. 2008; Nakamura et al. 2019). These results indicate that the development of cartilage and the occurrence of cartilage-related diseases are regulated by miRNAs.

Based on our previous findings, this study aimed to further explore cartilage development in PEE fetal rats at different time points of pregnancy. A three-dimensional chondroblast differentiation model of bone marrow mesenchymal stem cells (BMSCs) was used to simulate intrauterine articular cartilage development, and functional experiments were performed on GD17 primary chondrocytes to further investigate the underlying mechanism. We aimed to elucidate the specific time points of fetal chondroplasia induced by PEE and the molecular mechanism of ethanol-mediated fetal chondroplasia by upregulating miR-200b-3p expression and then inhibiting the ERG/PTHrP signaling pathway.

\section{Methods}

\subsection{Reagents}

Ethanol was purchased from Zhen Xin Co. Ltd. (Shanghai, China). Isoflurane was provided by Baxter Healthcare Co. (Deerfield, IL, USA). TRIzol reagent kits were obtained from Omega Bio-Tek (Doraville, USA). The RNA-Solv reagent and HiBindTM PCR DNA extraction kit were obtained from Omega Bio-Tek Inc. (Norcross, GA, USA). Primary antibodies against Col2a1 (ab34712), ERG (ab214341), and PTHLH (ab239527) were purchased from Abcam (Cambridge, UK); anti-Col10a1 (bs-0554R-FITC) obtained from Biosynthesis Biotechnology Co., Ltd. (Beijing, China). Real-time qPCR (RT-PCR) kits were purchased from TaKaRa Biotechnology Co., Ltd. (Dalian, China). All oligonucleotide primers specific to rat genes were synthesized by Sangon Biotech Co., Ltd. (Shanghai, China).

\subsection{Animals and treatment}

Specific pathogen-free (SPF) Wistar rats (females weighing 210-250 g, and males weighing 270-320 g) were purchased from the Experimental Center of Hubei Medical Scientific Academy (No. 2015-2018, certification number: 42000600032526, Wuhan, China), and animal handling and experimental procedures were carried out with approval from the Institute of Health Sciences Institutional Animal Care and Use Committee. All rats were fed adaptively for 1 week, and 2 females were mated with 1 male every night. The day when sperm was found on a vaginal smear was considered GD 0 . Then, the pregnant rats were randomly separated into two groups: the control group and the PEE group ( $n=24$ in each group). 
The PEE group was administered $4 \mathrm{~g} / \mathrm{kg} \cdot \mathrm{d}$ ethanol by gavage from GD9 to GD20, while the control group was administered the same volume of distilled water. Eight randomly selected pregnant rats from each group were anesthetized with isoflurane and decapitated on GD14, GD17, and GD20. At these three time points, one fetal rat was randomly selected from each litter and fixed with $95 \%$ alcohol for subsequent skeleton analysis. The knee joints of the remaining rats in the two groups were removed under an anatomical microscope, the right limbs were fixed in a $4 \%$ paraformaldehyde (PFA) solution, and the left limbs were stored in a $-80{ }^{\circ} \mathrm{C}$ freezer for gene detection. The scheme of animal experimental procedures was as follows.

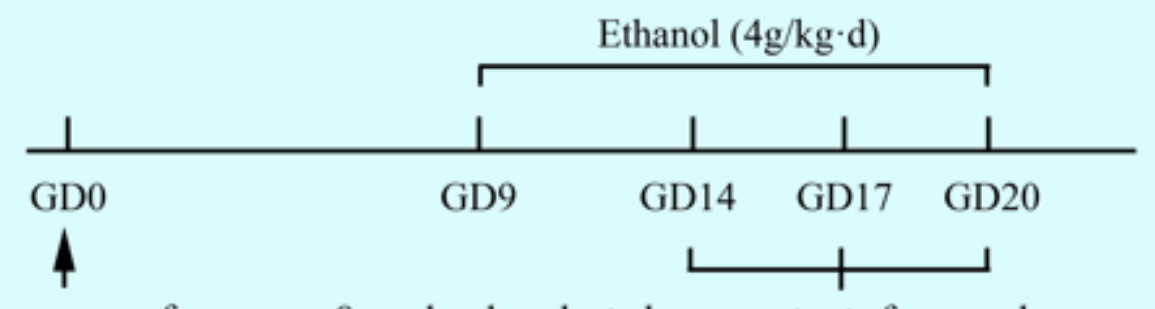

The appearance of sperm 8 randomly selected pregnant rats from each group were euthanized

in a vaginal smear and fetal cartilage was collected at each time

\subsection{Whole-mount skeletal staining}

Whole-mount skeletal staining using Alcian blue and Alizarin red was performed as described previously(Singh et al. 2018). Rat embryos of GD17 and GD20 were collected, rinsed in PBS and fixed in $95 \%$ ethanol for three days, followed by overnight fixation in 100\% acetone. Then, the tissues were stained for three days in a 1:1:1:17 volume mixture of glacial acetic acid:0.3\% Alcian blue 8GX (SigmaAldrich) in 95\% ethanol:0.1\% Alizarin red in 70\% ethanol:70\% ethanol. After staining, the tissues were washed using $1 \% \mathrm{KOH}$ and imaged under a Canon EOS 70D camera (Canon, Tokyo, Japan).

\subsection{Histological measurement}

Rat embryonic limbs on GD14, GD17, and GD20 were dissected, fixed in 4\% PFA at $4^{\circ} \mathrm{C}$ for three days, and embedded in paraffin, and $4 \mu \mathrm{m}$ sections were cut along the parasagittal plane using a microtome. For histological analysis, Alcian blue and safranin 0 were applied to detect the glycosaminoglycan (GAG) content. Histological images were captured using a Nikon NIS Elements BR light microscope (Nikon, Tokyo, Japan).

Immunohistochemical staining and tissue immunofluorescence staining were performed following the manufacturer's protocol. Briefly, after dewaxing, EDTA containing antigen retrieval buffer ( $\mathrm{pH} \mathrm{8.0)}$ was used for antigen retrieval. BSA was used to block the previously added primary antibody, and the primary antibody dilution ratios were as follows: anti-Col2a1 (1:200 dilution), anti-Col10a1 (1:200 dilution), antiPTHrP (1:250 dilution), and anti-ERG (1:250 dilution). Immunohistochemistry was conducted using a DAB staining kit (GeneTech Company, Ltd., Shanghai, China). For tissue immunofluorescence, the primary antibody was detected with a fluorescent Cy3-conjugated goat anti-rabbit lgG $(H+L)$ (1:50 dilution) 
secondary antibody, after which the tissue sections were stained with 4', 6-diamidino-2-phenylindole (DAPI) and sealed with an anti-fluorescence quenching agent. All images were captured and then analyzed with a Nikon NIS Elements BR light microscope (Nikon, Tokyo, Japan). The staining intensity was calculated by measuring the mean integrated optical density (MOD) in 10 different fields for each sample.

\subsection{Bioinformatics analysis}

The miRNAs targeting ERG were predicted according to three bioinformatics programs, miRDB (http://mirdb.org/), TargetScan (http://www.targetscan.org/vert_71/), and microRNA.org (http://www.microrna.org). The overlapping miRNAs are shown in the Venn diagram.

\subsection{Chondrogenic differentiation of BMSCs in alginate bead culture}

The extraction of BMSCs and their culture in alginate beads were conducted as described in a previous study(De Ceuninck et al. 2004; Deng et al. 2012). Monolayer cultures were trypsinized, washed, and centrifuged. The isolated BMSCs were suspended at a concentration of $1 \times 10^{7}$ cells $/ \mathrm{ml}$ in $1.25 \%$ alginate (Sigma-Aldrich, USA) in $0.15 \mathrm{M} \mathrm{NaCl}$. The cell suspension was drawn into a syringe and slowly added to a $10^{2} \mathrm{mM}$ calcium chloride solution dropwise with a needle. Beads with approximately $5 \times 10^{5} \mathrm{cells} / \mathrm{bead}$ were cultured in chondrogenic medium, containing high-glucose DMEM supplemented with $1 \%$ insulin, transferrin, and selenous acid (ITS) (Sigma-Aldrich, USA), $100 \mathrm{nM}$ dexamethasone (Sigma-Aldrich, USA), $50 \mu \mathrm{g} / \mathrm{ml}$ ascorbic acid-2-phosphate (Sigma-Aldrich, USA), $40 \mu \mathrm{g} / \mathrm{ml}$ I-proline (Sigma-Aldrich, USA), and $10 \mathrm{ng} / \mathrm{ml}$ transforming growth factor- $\beta 1$ (TGF- $\beta 1$ ) (PeproTech, USA). After chondrogenic differentiation for 3 weeks, alginate bead sections were stained with safranin- 0 to assess the number of cells and to locate GAG deposits. During the period of chondrogenic differentiation, the culture medium with or without ethanol at concentrations of $0,30,60$, and $120 \mathrm{mM}$ was replaced every other day.

\subsection{Primary chondrocyte culture}

Chondrocytes were isolated from GD17 rats and plated at a density of $2 \times 10^{5}$ cells per well in 6-well plates in medium (DMEM/F12 medium supplemented with $10 \%$ fetal bovine serum, $100 \mathrm{mg} / \mathrm{ml}$ streptomycin, and $100 \mathrm{U} / \mathrm{ml}$ penicillin). Primary chondrocytes at $80 \%$ confluence were used for further experiments. During the culture period, the cells were incubated at $37^{\circ} \mathrm{C}$ in a humidified atmosphere of $5 \%$ $\mathrm{CO}_{2}$ and $95 \%$ air.

\subsection{Transfection}

ERG and PTHLH overexpression plasmids were produced by Shanghai GenePharma Co., Ltd. (Shanghai, China). The miR-200b-3p inhibitor and mimic were synthesized by RiboBio (Guangzhou, China).

Subconfluent GD17 rat primary chondrocytes in 6-well plates were transfected in triplicate with $2.5 \mu \mathrm{g}$ of ERG and PTHLH overexpression plasmids using Lipofectamine ${ }^{\mathrm{TM}} 3000$ and $\mathrm{P} 3000^{\mathrm{TM}}$ transfection reagent (Invitrogen, USA) according to the manufacturer's protocol. For miRNA inhibitor and mimic transfection, 
cells were transfected with $100 \mathrm{nM}$ miR-200b-3p inhibitor or $50 \mathrm{nM}$ miR-200b-3p mimic using Lipofectamine $^{\mathrm{TM}} 3000$ transfection reagent (Invitrogen, USA) according to the manufacturer's protocol. After 8 hours, the transfection efficiency was determined using a fluorescence microscope. After 24 and 48 hours, the cells were collected for further analysis of gene and protein levels.

\subsection{Cellular immunofluorescence staining}

After treatment, the cells cultured on coated plates were washed three times with PBS, fixed in $4 \%$ formaldehyde for $15 \mathrm{~min}$, blocked with 3\% BSA for 1 hour, and permeabilizes with $0.1 \%$ Triton X-100/PBS for $10 \mathrm{~min}$. The cells were then incubated overnight at $4^{\circ} \mathrm{C}$ with primary antibodies in $0.5 \% \mathrm{BSA}$, including rabbit anti-Col2a1 (1:200 dilution), rabbit anti-Col10a1 (1: 50 dilution), mouse anti-ERG (1: 100 dilution), and mouse anti-PTHrP (1: 100 dilution). The cells were washed with PBS and incubated with 1:200 diluted Cy3-conjugated goat anti-rabbit and Fluor 594-conjugated goat anti-mouse secondary antibodies for 1 hour at room temperature. The nuclei were stained with DAPI at a 1:500 dilution for $5 \mathrm{~min}$. The slides were washed twice with PBS, and fluorescence images were captured using a confocal microscope (Smartproof 5, Carl Zeiss, Oberkochen, Germany). The staining intensity was determined by measuring the IOD in 10 different fields for each sample.

\subsection{Western blotting}

Briefly, resuspended cells were rinsed with ice-cold PBS and then lysed for $30 \mathrm{~min}$ at $4^{\circ} \mathrm{C}$ in RIPA lysis buffer containing phosphatase inhibitor cocktail, followed by analysis with the BCA Assay Kit for protein quantification. A total of $20 \mu \mathrm{g}$ of protein was loaded into each lane, separated by SDS-PAGE, and blotted onto PVDF membranes (Millipore, MA, USA). The membranes were blocked in $5 \%$ nonfat milk for 1 hour and incubated overnight at $4^{\circ} \mathrm{C}$ with the primary antibody. The dilution concentrations of the primary antibodies were as follows: Col2a1 (1:1000), Col10a1 (1:800), PTHrP (1:3000), and ERG (1: 3000). Then, the blots were incubated with horseradish peroxidase (HRP)-conjugated secondary antibody (goat antirabbit IgG, 1:5000 and goat anti-mouse IgG, 1:5000) for 1.5 hours and visualized using ECL HRP substrate (PerkinElmer Inc., Boston, MA, USA). The antibody binding signals were detected using a ChemiDoc Image Analyzer (Bio-Rad, Hercules, CA, USA). The relative protein level was standardized to the GAPDH protein level. The protein band intensities were analyzed by ImageJ (National Institutes of Health, Bethesda, MD, USA) from 3 independent bands.

\subsection{Total RNA extraction and RT-PCR}

Total RNA was isolated from cartilage tissues and primary chondrocytes using TRIzol reagent following the manufacturer's protocol. The RT-PCR procedure was described by Li et al.(Qing-Xian et al. 2020). RNA was assayed for GAPDH, Col2a1, aggrecan, tenascin-C, Col10a1, PTHLH, and ERG espression. All primers were designed with Primer Premier 6.0 (Premier Biosoft International, Palo Alto, CA, USA). The primer sequences for the rat genes are shown in Table 1. GAPDH served as the reference gene to normalize the expression of other genes. The relative expression levels of target genes were calculated by the $2^{-\Delta \Delta C t}$ method. 


\subsection{Luciferase reporter assay}

Lipofectamine $^{\text {TM }} 3000$ reagent was used to transiently transfect reporter plasmids into chondrocytes. For the dual luciferase assay, $6.0 \times 10^{4}$ chondrocytes in a 24-well plate were transfected with $100 \mathrm{nM}$ SV40ERG-WT or SV40-ERG-MUT. The chondrocytes were then cotransfected with $50 \mathrm{nM}$ miR-200b-3p mimic or negative control (NC). After incubation for $48 \mathrm{~h}$, the transfected cells were lysed and luciferase activity was detected using the Dual-Luciferase ${ }^{\circledR}$ Reporter Assay System (Promega, Madison, WI, USA). Firefly luciferase activity was normalized to Renilla luciferase activity. Three biological replicates were used for each experiment, and each experiment was repeated five times.

\subsection{Statistical analysis}

Prism Graphics (GraphPad Software, La Jolla, CA, USA, version 8.0) was applied for all data analyses. Quantitative data are expressed as the mean \pm S.D. The data of in vitro experiments with different ethanol concentrations were analyzed using one-way analysis of variance (ANOVA) with a post hoc test for multiple comparisons. For the data from the in vivo study, unpaired, two-tailed Student's t-tests were conducted for comparisons between the control and PEE groups. Correlation analysis of gene expression with and cartilage phenotype was performed using the Pearson correlation coefficient. Statistical significance was considered at $P<0.05$ for all the tests.

\section{Results}

\subsection{Skeletal and limb development of PEE fetal rats at different times during pregnancy}

To determine the effect of PEE on cartilage development, we examined the skeletal conditions of the fetal rats at different times (GD14, GD17, and GD20) (Fig. 1a). We found that the body weight, body length, femur length, and tibia length of PEE fetal rats were significantly lower than those of control rats on GD17 and GD20, but there was no significant difference between the two groups on GD14 (Fig. 1b). Additionally, the growth ratios of body weight, body length, femur length, and tibia length between the PEE group and the control group were 1.52, 1.3, 1.41, and 1.3, respectively, during GD14-17, while the growth ratios of the two groups during GD17-20 were 1.04, 0.99, 1.09, and 1.09, respectively. These results suggest that PEE can reduce the body weight and shorten the skeleton length of fetal rats in utero, especially during GD14-17.

On GD14, Alcian blue staining showed that the interzone formed in both groups was similar, and the articular cavity had not yet formed in either groups (Fig. 1c), indicating that there was no difference in cartilage development between the two groups during joint formation. On GD17, the articular cavity had formed in the control group, while it was not fully formed in the PEE group (Fig. 1c), indicating that the formation of articular cartilage was blocked in the PEE group. In addition, compared with that in the 
control group, the range of the proliferative zone (PZ) in the PEE group was significantly reduced, while the range of the hypertrophic zone ( $\mathrm{HZ}$ ) was significantly increased (Fig. 1d). On GD20, the range of the $\mathrm{PZ}$ and the primary ossification center (POC) in the PEE group was noticeably smaller than that in the control group, while the $\mathrm{HZ}$ in the PEE group was remarkably larger than that in the control group (Fig. $1 \mathrm{e})$. These results suggest that PEE can cause intrauterine limb dysplasia, mainly during the stage of articular cartilage formation (GD14-17), including narrowing of the cartilage proliferation area, delay in POC formation, and widening of the cartilage hypertrophy area.

\subsection{Morphology of local cartilage and expression of marker genes in fetal rats with PEE at different times during pregnancy}

We observed the morphology of the local cartilage at different times during pregnancy (GD14, GD17, and GD20). On GD14, condensation initially displayed no overt morphological signs of the knee or elbow joint, as observed by Safranin 0 staining. Moreover, there was no significant difference in the optical density of GAG or the number of chondrocytes per unit area in the knee joint between the two groups (Fig. 2a and $2 b)$. Additionally, by observing the cartilage development of the ankle joint and toe of GD14 fetal rats, we found that the cartilage staining of the two groups was consistent, and there was no significant difference in the number of chondrocytes per unit area (Supplementary Fig. 1). On GD17, compared with that of the control group, the GAG content in the knee joint cartilage of the PEE group was decreased, and the number of chondrocytes per unit area in the knee joint area was significantly reduced (Fig. 2a and 2b). Similarly, we found consistent changes in the elbow joints of fetal rats (Supplementary Fig. 2). On GD20, the development of articular cartilage in the PEE group was significantly slower than that in the control group (Fig. 2a and 2b). In summary, PEE can slow the development of intrauterine cartilage.

To more thoroughly examine the effect of PEE on cartilage, RT-qPCR was used to quantify the expression of cartilage function genes. The results showed that the mRNA levels of tenascin- $C$, aggrecan, and Col2a1 on GD17 and GD20 in the PEE group were significantly lower than those in the control group, while there was no significant difference between the two groups on GD14 (Fig. 2c). Additionally, during GD14-17, the change ratios of tenascin-C, aggrecan, Col2a1 and Col10a1 mRNA expression between the control group and PEE group were 2.74, 5.3, 1.97, and 0.48, respectively, while during GD17-20, the change ratios were $1.1,1.11,1.13$, and 0.95 , respectively. These findings suggest that the toxic effect of PEE on cartilage development mainly occurs on GD14-17. The results of tissue immunofluorescence of Col2a1 and Col10a1 were consistent with those of RT-qPCR (Fig. 2d-2f). Overall, PEE reduced the expression of genes associated with fetal articular cartilage development and increased the expression of hypertrophic genes, which mainly occured in the stage of articular cartilage formation.

\subsection{Expression of miR-200b-3p, ERG, and PTHrP in PEE fetal rats at different times during pregnancy}


To explore the mechanism of cartilage dysplasia induced by PEE, we detected the expression of cartilage regulatory genes, including ERG and PTHrP. The results of immunohistochemistry showed that the expression of ERG and PTHrP in the cartilage of PEE offspring was significantly lower than that in the control offspring on GD17 and GD20, but there was no significant difference between the two groups on GD14 (Fig. 3a-c). RT-qPCR showed the same results (Fig. 3d). Moreover, the change ratio of ERG and PTHrP mRNA expression levels between the control group and the PEE group was 3.7 and 2.95, respectively, in the articular cartilage formation stage (GD14-17); however, during GD17-20, the change ratio of the two groups was 1.03 and 1.11, respectively. There results suggest that PEE has a significant effect on the formation of articular cartilage. Furthermore, we found that the mRNA expression of ERG and PTHrP on GD17 was positively correlated with articular cartilage phenotypes, such as the content of articular cartilage matrix (GAG) and the number of chondrocytes per unit area (Fig. 3e), and negatively correlated with cartilage hypertrophy phenotypes, such as the mRNA expression of Col10a1 and HZ/PZ (Fig. 3f). We obtained the same results in GD20 fetal cartilage (Supplementary Fig. 3). These results indicate that the expression of ERG and PTHrP in fetal cartilage is downregulated by PEE, which is related to the decrease in articular cartilage differentiation and the increase in cartilage hypertrophy.

Furthermore, we investigated the possible miRNAs targeting ERG by overlapping the predicted results of the miRDB, TargetScan, and microRNA.org databases (Supplementary Table 1). The common miRNAs are shown in the Venn diagram and include rno-miR-144-3p, rno-miR-139-5p, rno-miR-9a-3p, rno-miR200b-3p and rno-miR-200c-3p (Fig. 3g). Then, we detected the expression of these miRNAs in cartilage tissue, which showed that only the expression of rno-miR-200b-3p in the PEE group was significantly higher than that in the control group in both GD17 and GD20 samples (Fig. 3h). These results suggest that the high expression of miR-200b-3p may mediate the development of articular cartilage by inhibiting the expression of ERG and PTHrP in fetal rats induced by PEE.

\subsection{Effect of ethanol on chondroblast differentiation of BMSCs and the expression of the miR-200b-3p/ERG/PTHrP axis}

To verify the effect and mechanism of ethanol on cartilage development, we used a BMSC threedimensional culture model to simulate intrauterine cartilage development (Supplementary Fig. 4) and found that different concentrations of ethanol had no cytotoxicity on the differentiation of BMSCs (Supplementary Fig. 5). Safranin 0 staining showed that compared with that of the control group, the staining of groups treated with different concentrations of ethanol was significantly lighter, and the number of chondrocytes per unit area was reduced in a concentration-dependent manner (Fig. 4a-c). RTqPCR analysis showed that the mRNA expression of tenascin-C, aggrecan, and Col2a1 in groups treated with different concentrations of ethanol was significantly decreased, and the mRNA expression of Col10a1 was significantly increased in a concentration-dependent manner (Fig. 4d-g). Moreover, compared with that in the control group, the expression of miR-200b-3p was significantly increased and the mRNA expression of ERG and PTHrP was significantly decreased in the ethanol group (Fig. 4h-j). The protein levels of Col2a1, Col10a1, ERG, and PTHrP were consistent with the mRNA expression levels, as 
observed by western blotting (Fig. 4k-I). These results indicate that ethanol inhibited BMSCs from differentiating into articular cartilage in a concentration-dependent manner, promoted cartilage hypertrophy, increased the expression of miR-200b-3p, and decreased the expression of ERG and PTHrP.

\subsection{Regulatory effect of ERG/PTHrP on the differentiation of articular chondrocytes}

To assess the involvement of ERG/PTHrP in the regulation of ethanol-induced differentiation of articular chondrocytes, we transfected GD17 primary chondrocytes with PTHLH and ERG overexpression plasmids. Immunofluorescence at 8 hours after transfection confirmed that the PTHLH and ERG plasmids were successfully transfected (Supplementary Fig. 6). As shown in Fig. 5a, ethanol decreased the expression of tenascin- $\mathrm{C}$, aggrecan, and Col2a1 and promoted the espression of Col10a1, while the overexpression of PTHLH reversed these changes, as observed by RT-qPCR. Western blotting and immunofluorescence also confirmed that overexpression of PTHLH could reverse the effect of ethanol on the protein levels of Col2a1 and Col10a1 (Fig. 5b). Similarly, overexpression of ERG in GD17 primary chondrocytes could reverse ethanol-induced changes in both the mRNA level (Fig. 5d) and protein level of marker genes (Fig. 5e-f). Additionally, overexpression of ERG could reverse the low expression of PTHrP induced by ethanol, but overexpression of PTHLH could not reverse the effect of ethanol on PTHrP expression (Fig. 5g-h). These results suggest that ERG regulates the differentiation of articular chondrocytes by acting through PTHrP.

\subsection{Regulation of ERG and articular chondrocyte differentiation by miR-200b-3p}

To further explore whether miR-200b-3p is involved in the regulation of ERG by ethanol, we designed a luciferase reporter assay (Fig. 6a). Our data showed that the miR-200b-3p mimic significantly inhibited luciferase activity in cells transfected with SV40-ERG-WT but not in those transfected with SV40-ERGMUT (Fig. 6b). Therefore, miR-200b-3p can target and negatively regulate ERG. RT-PCR, western blotting, and immunofluorescence further confirmed that $60 \mathrm{mM}$ ethanol significantly decreased the mRNA and protein levels of ERG, while the miR-200b-3p inhibitor could partially reverse the inhibitory effect of ethanol on the expression of ERG; in the NC + miR-200b-3p inhibitor group, the mRNA and protein expression of ERG was significantly increased compared with that in the control group (Fig. 6c-e).

In addition, the mRNA expression of tenascin-C, aggrecan, and Col2a1 was markedly reduced when the cells were exposed to $60 \mathrm{mM}$ ethanol, and the mRNA expression of Col10a1 was dramatically upregulated; however, the miR-200b-3p inhibitor reversed the above changes (Fig. 6f). Moreover, western blotting and immunofluorescence results showed that the miR-200b-3p inhibitor could reverse the effect of ethanol on the protein levels of Col2a1 and Col10a1 (Fig. 6g-h). Overall, these lines of evidence suggest that miR-200b-3p negatively regulates the expression of ERG and partially reverses the decrease in ERG expression and articular cartilage dysplasia induced by ethanol.

\section{Discussion}




\subsection{Articular cartilage dysplasia in PEE offspring originated from the second trimester}

In the past 60 years, people have been aware of the harm of alcohol exposure during pregnancy, but alcohol intake during pregnancy is still an important public health problem(Vall et al. 2015). It has been reported that $5.8 \%$ of women in Canada and approximately $12.5 \%$ of women in the USA drink alcohol during pregnancy(Delano et al. 2019). In China $26.9 \%$ of adult women drink alcohol, and $0.2 \%$ of them are affected by alcohol dependence(Centers for Disease and Prevention 2009). Previous studies have found that offspring with PEE have characteristic craniofacial deformities, low birth weight and limb shortening, that the effect of alcohol on fetal bone development is difficult to correct after birth, and that high-dose alcohol exposure during pregnancy leads to life-long limb shortening in offspring(Day et al. 2002; Keiver and Weinberg 2004). In this study, we found that, consistent with the studies of Snow(Snow and Keiver 2007) and Pan(Pan et al. 2016), PEE could induce hypertrophic transition cartilage, increase the length of the hypertrophic cartilage area and increase the expression of the hypertrophy marker gene Col10a1; however, limb length and POC were short, which shows the inhibition of osteogenic differentiation. These differences were most prominent at GD17 and GD20, which showed that the weight, body length, femur length and tibia length of PEE fetal rats were significantly lower than those of control rats. This is related to the inhibition of the terminal differentiation of transitional cartilage by PEE.

Interestingly, in this study, we also found that PEE can cause dysplasia of articular cartilage, which shares a common tissue source with transitional cartilage. The theory that adult OA has intrauterine origin was first proposed and proven by our previous research(Chen et al. 2019a; Li et al. 2020; Ni et al. 2015a; Ni et al. 2018; Ni et al. 2015b; Pan et al. 2016; Tie et al. 2016a; Tie et al. 2016b). In this study, we dynamically observed the effects of ethanol on articular cartilage development in fetuses at three time points: arthrogenesis (GD14), articular chondrogenesis (GD17) and just before birth (GD20). We found that on GD14, ethanol did not significantly affect the development of articular cartilage in offspring, while on GD17 and GD20, the development of articular cartilage in the ethanol group was significantly delayed compared with that in the control group. The growth rate analysis showed that the growth rate of body length and limb length from GD14 to GD17 in the control group was significantly higher than that in the PEE group, but there was no significant difference between the two groups from GD17 to GD20. Moreover, compared with those in the control group, the change rates of expression levels of the cartilage marker genes COL2A1 and Col10a1, as well as the cartilage development regulation genes ERG and PTHrP, in PEE group were significantly different from GD14 to GD17, while there was no significant difference between the two groups from GD17 to GD20. These results indicate that the toxicity of PEE to fetal cartilage mainly occurs in the period of articular cartilage formation, i.e., GD14-17, rather than in GD17-20, indicating that there is a sensitive period (articular cartilage formation stage) for the toxic effect of PEE on cartilage development, rather than a cumulative toxic effect of PEE. Previous studies also suggested that the cartilage progenitor cells in this period had strong plasticity, and external factors could induce them to differentiate into articular cartilage or hypertrophic cartilage(Kahn et al. 2009; Ray et al. 2015). We used the chondrogenic differentiation model of BMSCs to simulate the process of intrauterine 


\subsection{ERG/PTHrP mediated articular chondrodysplasia in PEE fetal rats in the middle and late stages of pregnancy}

ERG is initially expressed in the mesenchymal interzone, and expression continues in epiphyseal articular chondrocytes over the developmental period(Iwamoto et al. 2007). Studies have shown that ERG is an important component of the synovial joint formation mechanism that can regulate the developmental behavior of most epiphyseal chondrocytes and help them acquire the permanent phenotypes of articular chondrocytes(Iwamoto et al. 2001; Iwamoto et al. 2007). Singh et al. found that, together with NFIA and GDF5, ERG could inhibit the differentiation of transient cartilage in articular cartilage. ERG and PTHrP can also control the crosstalk between articular cartilage differentiation and hypertrophy(Sathyan et al. 2007; Singh et al. 2018). In this study, it was found that at GD17 and GD20, the mRNA and protein expression levels of ERG and PTHrP in the PEE group were significantly lower than those in the control group, and the expression levels of ERG and PTHrP were positively correlated with the articular cartilage phenotype and negatively correlated with the cartilage hyperplasia phenotype. At GD20, POC was formed in both groups, but the length of POC in the PEE group was significantly shorter than that in the control group. Meanwhile, the $\mathrm{HZ}$ region adjacent to the $\mathrm{POC}$ and the $\mathrm{PZ}$ region in the metaphysis of the PEE group were also significantly shorter than those in the control group. Similar to the changes in ERG expression, PTHrP mRNA expression in the PEE group was significantly lower than that in the control group at GD17 and GD20. Overexpression of PTHLH or ERG reversed the inhibitory effect of ethanol on the expression of articular cartilage marker genes in GD17 primary chondrocytes, indicating that low expression of ERG and PTHrP mediates ethanol-induced dysplasia of articular cartilage. In addition, the present study found that overexpression of ERG could reverse the low expression of PTHrP induced by ethanol, but overexpression of PTHLH could not reverse the low expression of ERG induced by ethanol, suggesting that ERG functions by regulating the expression of PTHrP. Previous studies also have confirmed that ERG can regulate chondrocyte development by regulating the expression of PTHrP under physiological conditions(Ohta et al. 2015). Consistent with Okabe's results(Okabe et al. 2011), overexpression of ERG promoted the expression of PTHrP. These results suggest that the low expression of ERG and PTHrP mediates ethanolinduced fetal articular cartilage dysplasia. Therefore, we propose that ERG/PTHrP mediates PEE-induced fetal articular chondrodysplasia.

\subsection{Upregulation of miR-200b-3p expression by ethanol and targeted regulation of ERG induced progeny articular cartilage dysplasia}


miRNAs play an important role in development and are associated with many birth defects. New evidence suggests that miRNAs are sensitive to ethanol during development and mediate ethanol-induced teratogenesis(Miranda et al. 2010; Sathyan et al. 2007; Wang et al. 2009). In addition, a large number of studies have found that miR-200 plays an important role in a variety of human malignancies(Miranda et al. 2010; Sathyan et al. 2007; Wang et al. 2009). Recent studies have confirmed that miR-200b-3p is involved in articular cartilage degeneration and plays an important role in the progression of OA(Wu et al. 2017). Interestingly, the latest research by $\mathrm{MOH} \mathrm{MOH}$ Aung shows that miR-200b-3p can downregulate the expression of ERG and inhibit vascular proliferation in endothelial cells(Moh-Moh-Aung et al. 2020). In this study, we found that the expression of miR-200b-3p in fetal cartilage of PEE rats was significantly upregulated and negatively correlated with the expression of ERG. We further confirmed that miR-200b-3p could directly target and negatively regulate the expression of ERG, as confirmed by the luciferase reporter assay. In addition, a miR-200b-3p inhibitor reversed the inhibitory effect of ethanol on ERG and chondrogenic differentiation. Overall, we can conclude that PEE can upregulate the expression of miR$200 \mathrm{~b}-3 \mathrm{p}$ in local cartilage and further suppress the expression of ERG, thereby inhibiting the differentiation of cartilage progenitor cells into articular chondrocytes and causing articular chondrodysplasia.

\section{Summary}

In conclusion, this study confirmed the existence of articular cartilage dysplasia in PEE offspring, which mainly occurred in the period of articular cartilage formation, namely, GD14-17. The mechanism is related to the decreased differentiation of chondrogenic progenitor cells into articular chondrocytes due to the upregulation of miR-200b-p expression by ethanol and subsequent downregulation of PTHrP expression by targeted inhibition of ERG. In this study, for the first time, the development of cartilage at different stages of intrauterine development was detected, and the specific time course and mechanism of articular chondrodysplasia induced by ethanol were clarified, providing a more accurate and in-depth theoretical basis for the analysis of the developmental toxicity of PEE in fetal cartilage.

\section{Declarations}

Acknowledgments: This work was supported by grants from the National Natural Science Foundation of China (No. 81803275, 81603214, 81673490, 81673524, 81972036), and the Key Research and Development Project of Hubei province (No. 2020BCA071).

Funding: This work was supported by grants from the National Natural Science Foundation of China (No. 81803275, 81603214, 81673490, 81673524, 81972036), and the Key Research and Development Project of Hubei province (No. 2020BCA071).

Conflicts of interest Statement: All the authors state that they have no conflicts of interest. 
Ethics approval $₫$ All animal experimental procedures were conducted in accordance with the Guidelines for the Care and Use of Laboratory Animals of the Chinese Animal Welfare Committee.

Consent to participate: Not applicable.

Consent for publication: All authors agree that this article will be published in Cell Biology and Toxicology.

Availability of data and material: The data that support the findings of this study are available from the corresponding author upon reasonable request.

Code availability: Not applicable

Author Contributions: Qubo Ni, Haitao Chen and Bin Li contributed to the acquisition of animal data. Qubo Ni and Haitao Chen performed the RNA isolation and participated in the experimental design, interpretation and manuscript writing. Hangyuan He and Jiayong Zhu assisted with cell experiment, data analysis and interpretation of findings. Hangyuan He, Jiayong Zhu and Hui Gao provided critical revision of the manuscript for important intellectual content. Hui Wang and Liaobin Chen participated in the experimental design, the supervision, interpretation and revised the paper.

\section{References}

1. Abubakar, A.A., Noordin, M.M., Azmi, T.I., Kaka, U., and Loqman, M.Y. 2016. The use of rats and mice as animal models in ex vivo bone growth and development studies. Bone \& joint research 5(12): 610618. doi:10.1302/2046-3758.512.BJR-2016-0102.R2.

2. Aigner, T., and Richter, W. 2012. OA in 2011: Age-related OA--a concept emerging from infancy? Nature reviews. Rheumatology 8(2): 70-72. doi:10.1038/nrrheum.2011.206.

3. Barter, M.J., Tselepi, M., Gomez, R., Woods, S., Hui, W., Smith, G.R., et al. 2015. Genome-Wide MicroRNA and Gene Analysis of Mesenchymal Stem Cell Chondrogenesis Identifies an Essential Role and Multiple Targets for miR-140-5p. Stem cells 33(11): 3266-3280. doi:10.1002/stem.2093.

4. Blaker, C.L., Clarke, E.C., and Little, C.B. 2017. Using mouse models to investigate the pathophysiology, treatment, and prevention of post-traumatic osteoarthritis. Journal of orthopaedic research : official publication of the Orthopaedic Research Society 35(3): 424-439. doi:10.1002/jor.23343.

5. Bukowska, J., Frazier, T., Smith, S., Brown, T., Bender, R., McCarthy, M., et al. 2018. Bone Marrow Adipocyte Developmental Origin and Biology. Current osteoporosis reports 16(3): 312-319. doi:10.1007/s11914-018-0442-z.

6. Centers for Disease, C., and Prevention. 2009. Alcohol use among pregnant and nonpregnant women of childbearing age - United States, 1991-2005. MMWR. Morbidity and mortality weekly report 58(19): 529-532. Available from http://www.ncbi.nlm.nih.gov/pubmed/19478721 [accessed. 
7. Chen, B., Lu, K.H., Ni, Q.B., Li, Q.X., Gao, H., Wang, H., et al. 2019a. Prenatal nicotine exposure increases osteoarthritis susceptibility in male elderly offspring rats via low-function programming of the TGFbeta signaling pathway. Toxicology letters 314: 18-26. doi:10.1016/j.toxlet.2019.06.010.

8. Chen, S., Xu, Z., Shao, J., Fu, P., and Wu, H. 2019b. MicroRNA-218 promotes early chondrogenesis of mesenchymal stem cells and inhibits later chondrocyte maturation. BMC biotechnology 19(1): 6 . doi:10.1186/s12896-018-0496-0.

9. Coutinho de Almeida, R., Ramos, Y.F.M., Mahfouz, A., den Hollander, W., Lakenberg, N., Houtman, E., et al. 2019. RNA sequencing data integration reveals an miRNA interactome of osteoarthritis cartilage. Annals of the rheumatic diseases 78(2): 270-277. doi:10.1136/annrheumdis-2018-213882.

10. Day, N.L., Leech, S.L., Richardson, G.A., Cornelius, M.D., Robles, N., and Larkby, C. 2002. Prenatal alcohol exposure predicts continued deficits in offspring size at 14 years of age. Alcoholism, clinical and experimental research 26(10): 1584-1591. doi:10.1097/01.ALC.0000034036.75248.D9.

11. De Ceuninck, F., Lesur, C., Pastoureau, P., Caliez, A., and Sabatini, M. 2004. Culture of chondrocytes in alginate beads. Methods in molecular medicine 100: 15-22. doi:10.1385/1-59259-810-2:015.

12. Delano, K., Koren, G., Zack, M., and Kapur, B.M. 2019. Prevalence of Fetal Alcohol Exposure by Analysis of Meconium Fatty Acid Ethyl Esters; A National Canadian Study. Scientific reports 9(1): 2298. doi:10.1038/s41598-019-38856-5.

13. Deng, Y., Li, T.Q., Yan, Y.E., Magdalou, J., Wang, H., and Chen, L.B. 2012. Effect of nicotine on chondrogenic differentiation of rat bone marrow mesenchymal stem cells in alginate bead culture. Bio-medical materials and engineering 22(1-3): 81-87. doi:10.3233/BME-2012-0692.

14. Iwamoto, M., Koyama, E., Enomoto-Iwamoto, M., and Pacifici, M. 2005. The balancing act of transcription factors C-1-1 and Runx2 in articular cartilage development. Biochemical and biophysical research communications 328(3): 777-782. doi:10.1016/j.bbrc.2004.11.073.

15. Iwamoto, M., Higuchi, Y., Enomoto-Iwamoto, M., Kurisu, K., Koyama, E., Yeh, H., et al. 2001. The role of ERG (ets related gene) in cartilage development. Osteoarthritis and cartilage 9 Suppl A: S41-47. doi:10.1053/joca.2001.0443.

16. Iwamoto, M., Tamamura, Y., Koyama, E., Komori, T., Takeshita, N., Williams, J.A., et al. 2007. Transcription factor ERG and joint and articular cartilage formation during mouse limb and spine skeletogenesis. Developmental biology 305(1): 40-51. doi:10.1016/j.ydbio.2007.01.037.

17. Jordan, K.M., Syddall, H., Dennison, E.M., Cooper, C., and Arden, N.K. 2005. Birthweight, vitamin D receptor gene polymorphism, and risk of lumbar spine osteoarthritis. The Journal of rheumatology 32(4): 678-683. Available from http://www.ncbi.nlm.nih.gov/pubmed/15801025 [accessed.

18. Kahn, J., Shwartz, Y., Blitz, E., Krief, S., Sharir, A., Breitel, D.A., et al. 2009. Muscle contraction is necessary to maintain joint progenitor cell fate. Developmental cell 16(5): 734-743. doi:10.1016/j.devcel.2009.04.013.

19. Kang, D., Shin, J., Cho, Y., Kim, H.S., Gu, Y.R., Kim, H., et al. 2019. Stress-activated miR-204 governs senescent phenotypes of chondrocytes to promote osteoarthritis development. Science translational medicine 11(486). doi:10.1126/scitranslmed.aar6659. 
20. Keiver, K., and Weinberg, J. 2004. Effect of duration of maternal alcohol consumption on calcium metabolism and bone in the fetal rat. Alcoholism, clinical and experimental research 28(3): 456-467. doi:10.1097/01.alc.0000118312.38204.c5.

21. Kobayashi, T., Lu, J., Cobb, B.S., Rodda, S.J., McMahon, A.P., Schipani, E., et al. 2008. Dicer-dependent pathways regulate chondrocyte proliferation and differentiation. Proceedings of the National Academy of Sciences of the United States of America 105(6): 1949-1954. doi:10.1073/pnas.0707900105.

22. Kornak, U., and Mundlos, S. 2003. Genetic disorders of the skeleton: a developmental approach. American journal of human genetics 73(3): 447-474. doi:10.1086/377110.

23. Kronenberg, H.M. 2003. Developmental regulation of the growth plate. Nature 423(6937): 332-336. doi:10.1038/nature01657.

24. Lawrence, R.C., Felson, D.T., Helmick, C.G., Arnold, L.M., Choi, H., Deyo, R.A., et al. 2008. Estimates of the prevalence of arthritis and other rheumatic conditions in the United States. Part II. Arthritis and rheumatism 58(1): 26-35. doi:10.1002/art.23176.

25. Le, L., Niu, L., Barter, M.J., Young, D.A., Dalmay, T., Clark, I.M., et al. 2020. The role of microRNA-3085 in chondrocyte function. Scientific reports 10(1): 21923. doi:10.1038/s41598-020-78606-6.

26. Li, J., Xiao, H., Luo, H., Tan, Y., Ni, Q., He, C., et al. 2020. GR/HDAC2/TGFbetaR1 pathway contributes to prenatal caffeine induced-osteoarthritis susceptibility in male adult offspring rats. Food and chemical toxicology : an international journal published for the British Industrial Biological Research Association 140: 111279. doi:10.1016/j.fct.2020.111279.

27. Martinez, B., and Peplow, P.V. 2021. MicroRNAs as diagnostic and prognostic biomarkers of agerelated macular degeneration: advances and limitations. Neural regeneration research 16(3): 440447. doi:10.4103/1673-5374.293131.

28. Miranda, R.C., Pietrzykowski, A.Z., Tang, Y., Sathyan, P., Mayfield, D., Keshavarzian, A., et al. 2010. MicroRNAs: master regulators of ethanol abuse and toxicity? Alcoholism, clinical and experimental research 34(4): 575-587. doi:10.1111/j.1530-0277.2009.01126.x.

29. Miyaki, S., Sato, T., Inoue, A., Otsuki, S., Ito, Y., Yokoyama, S., et al. 2010. MicroRNA-140 plays dual roles in both cartilage development and homeostasis. Genes \& development 24(11): 1173-1185. doi:10.1101/gad.1915510.

30. Moh-Moh-Aung, A., Fujisawa, M., Ito, S., Katayama, H., Ohara, T., Ota, Y., et al. 2020. Decreased miR200b-3p in cancer cells leads to angiogenesis in HCC by enhancing endothelial ERG expression. Scientific reports 10(1): 10418. doi:10.1038/s41598-020-67425-4.

31. Nakamura, A., Rampersaud, Y.R., Nakamura, S., Sharma, A., Zeng, F., Rossomacha, E., et al. 2019. microRNA-181a-5p antisense oligonucleotides attenuate osteoarthritis in facet and knee joints. Annals of the rheumatic diseases 78(1): 111-121. doi:10.1136/annrheumdis-2018-213629.

32. Ni, Q., Tan, Y., Zhang, X., Luo, H., Deng, Y., Magdalou, J., et al. 2015a. Prenatal ethanol exposure increases osteoarthritis susceptibility in female rat offspring by programming a low-functioning IGF1 signaling pathway. Scientific reports 5: 14711. doi:10.1038/srep14711. 
33. Ni, Q., Lu, K., Li, J., Tan, Y., Qin, J., Magdalou, J., et al. 2018. Role of TGFbeta Signaling in Maternal Ethanol-Induced Fetal Articular Cartilage Dysplasia and Adult Onset of Osteoarthritis in Male Rats. Toxicological sciences : an official journal of the Society of Toxicology 164(1): 179-190. doi:10.1093/toxsci/kfy080.

34. Ni, Q., Wang, L., Wu, Y., Shen, L., Qin, J., Liu, Y., et al. 2015b. Prenatal ethanol exposure induces the osteoarthritis-like phenotype in female adult offspring rats with a post-weaning high-fat diet and its intrauterine programming mechanisms of cholesterol metabolism. Toxicology letters 238(2): 117125. doi:10.1016/j.toxlet.2015.07.017.

35. Ohta, Y., Okabe, T., Larmour, C., Di Rocco, A., Maijenburg, M.W., Phillips, A., et al. 2015. Articular cartilage endurance and resistance to osteoarthritic changes require transcription factor Erg. Arthritis \& rheumatology 67(10): 2679-2690. doi:10.1002/art.39243.

36. Okabe, T., Ohta, Y., Asai, S., Wakitani, S., Enomoto-Iwamoto, M., Pacifici, M., et al. 2011. 72 ERG AND PTHRP GENETICALLY COOPERATE TO MAINTAIN ARTICULAR CARTILAGE LONG-TERM FUNCTION. Osteoarthritis and cartilage.

37. Pacifici, M., Koyama, E., and Iwamoto, M. 2005. Mechanisms of synovial joint and articular cartilage formation: recent advances, but many lingering mysteries. Birth defects research. Part $C$, Embryo today : reviews 75(3): 237-248. doi:10.1002/bdrc.20050.

38. Pan, Z., Zhang, X., Shangguan, Y., Hu, H., Chen, L., and Wang, H. 2016. Suppressed osteoclast differentiation at the chondro-osseous junction mediates endochondral ossification retardation in long bones of Wistar fetal rats with prenatal ethanol exposure. Toxicology and applied pharmacology 305: 234-241. doi:10.1016/j.taap.2016.06.021.

39. Pelosi, M., Lazzarano, S., Thoms, B.L., and Murphy, C.L. 2013. Parathyroid hormone-related protein is induced by hypoxia and promotes expression of the differentiated phenotype of human articular chondrocytes. Clinical science 125(10): 461-470. doi:10.1042/CS20120610.

40. Pitsillides, A.A., and Beier, F. 2011. Cartilage biology in osteoarthritis-lessons from developmental biology. Nature reviews. Rheumatology 7(11): 654-663. doi:10.1038/nrrheum.2011.129.

41. Qing-Xian, L., Lin-Long, W., Yi-Zhong, W., Liang, L., Hui, H., Liao-Bin, C., et al. 2020. Programming changes in GLUT1 mediated the accumulation of AGEs and matrix degradation in the articular cartilage of female adult rats after prenatal caffeine exposure. Pharmacological research 151: 104555. doi:10.1016/j.phrs.2019.104555.

42. Ray, A., Singh, P.N., Sohaskey, M.L., Harland, R.M., and Bandyopadhyay, A. 2015. Precise spatial restriction of BMP signaling is essential for articular cartilage differentiation. Development 142(6): 1169-1179. doi:10.1242/dev.110940.

43. Razmara, E., Bitaraf, A., Yousefi, H., Nguyen, T.H., Garshasbi, M., Cho, W.C., et al. 2019. Non-Coding RNAs in Cartilage Development: An Updated Review. International journal of molecular sciences 20(18). doi:10.3390/ijms20184475.

44. Sathyan, P., Golden, H.B., and Miranda, R.C. 2007. Competing interactions between micro-RNAs determine neural progenitor survival and proliferation after ethanol exposure: evidence from an ex 
vivo model of the fetal cerebral cortical neuroepithelium. The Journal of neuroscience : the official journal of the Society for Neuroscience 27(32): 8546-8557. doi:10.1523/JNEUROSCI.1269-07.2007.

45. Sayer, A.A., Poole, J., Cox, V., Kuh, D., Hardy, R., Wadsworth, M., et al. 2003. Weight from birth to 53 years: a longitudinal study of the influence on clinical hand osteoarthritis. Arthritis and rheumatism 48(4): 1030-1033. doi:10.1002/art.10862.

46. Shapiro, F. 2001. Developmental Bone Biology. 3-128. doi:10.1016/b978-012638651-6/50002-2.

47. Shen, S., Wu, Y., Chen, J., Xie, Z., Huang, K., Wang, G., et al. 2019. CircSERPINE2 protects against osteoarthritis by targeting miR-1271 and ETS-related gene. Annals of the rheumatic diseases 78(6): 826-836. doi:10.1136/annrheumdis-2018-214786.

48. Singh, P.N.P., Yadav, U.S., Azad, K., Goswami, P., Kinare, V., and Bandyopadhyay, A. 2018. NFIA and GATA3 are crucial regulators of embryonic articular cartilage differentiation. Development 145(2). doi:10.1242/dev.156554.

49. Snow, M.E., and Keiver, K. 2007. Prenatal ethanol exposure disrupts the histological stages of fetal bone development. Bone 41(2): 181-187. doi:10.1016/j.bone.2007.04.182.

50. Suomi, S., Taipaleenmaki, H., Seppanen, A., Ripatti, T., Vaananen, K., Hentunen, T., et al. 2008. MicroRNAs regulate osteogenesis and chondrogenesis of mouse bone marrow stromal cells. Gene regulation and systems biology 2: 177-191. doi:10.4137/grsb.s662.

51. Swingler, T.E., Wheeler, G., Carmont, V., Elliott, H.R., Barter, M.J., Abu-Elmagd, M., et al. 2012. The expression and function of microRNAs in chondrogenesis and osteoarthritis. Arthritis and rheumatism 64(6): 1909-1919. doi:10.1002/art.34314.

52. Tie, K., Tan, Y., Deng, Y., Li, J., Ni, Q., Magdalou, J., et al. 2016a. Prenatal nicotine exposure induces poor articular cartilage quality in female adult offspring fed a high-fat diet and the intrauterine programming mechanisms. Reproductive toxicology 60: 11-20. doi:10.1016/j.reprotox.2015.12.010.

53. Tie, K., Zhang, X., Tan, Y., Deng, Y., Li, J., Ni, Q., et al. 2016b. Intrauterine low-functional programming of IGF1 by prenatal nicotine exposure mediates the susceptibility to osteoarthritis in female adult rat offspring. FASEB journal : official publication of the Federation of American Societies for Experimental Biology 30(2): 785-797. doi:10.1096/fj.15-274399.

54. Vall, O., Salat-Batlle, J., and Garcia-Algar, O. 2015. Alcohol consumption during pregnancy and adverse neurodevelopmental outcomes. Journal of epidemiology and community health 69(10): 927929. doi:10.1136/jech-2014-203938.

55. Wang, L.L., Zhang, Z., Li, Q., Yang, R., Pei, X., Xu, Y., et al. 2009. Ethanol exposure induces differential microRNA and target gene expression and teratogenic effects which can be suppressed by folic acid supplementation. Human reproduction 24(3): 562-579. doi:10.1093/humrep/den439.

56. Wu, J., Tao, Y., Shang, A., Wang, W., Zhang, Y., Hu, L., et al. 2017. Effect of the interaction between MiR-200b-3p and DNMT3A on cartilage cells of osteoarthritis patients. Journal of cellular and molecular medicine 21(10): 2308-2316. doi:10.1111/jcmm.13152.

\section{Tables}




\section{Figures}

a
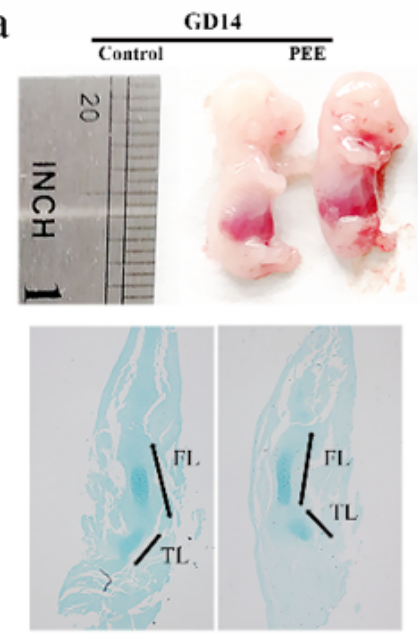

$\mathrm{c}$

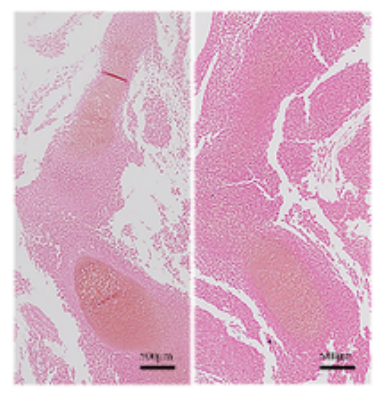

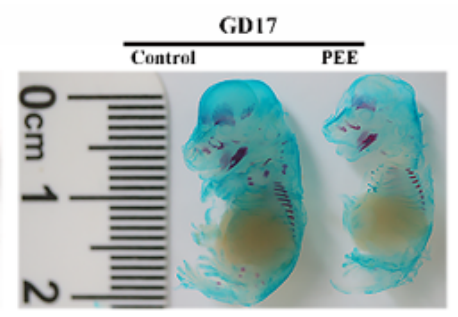
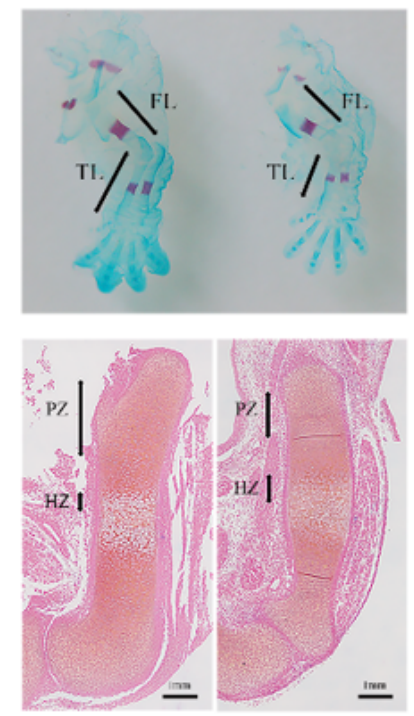

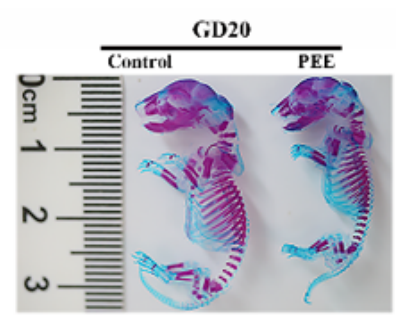

b
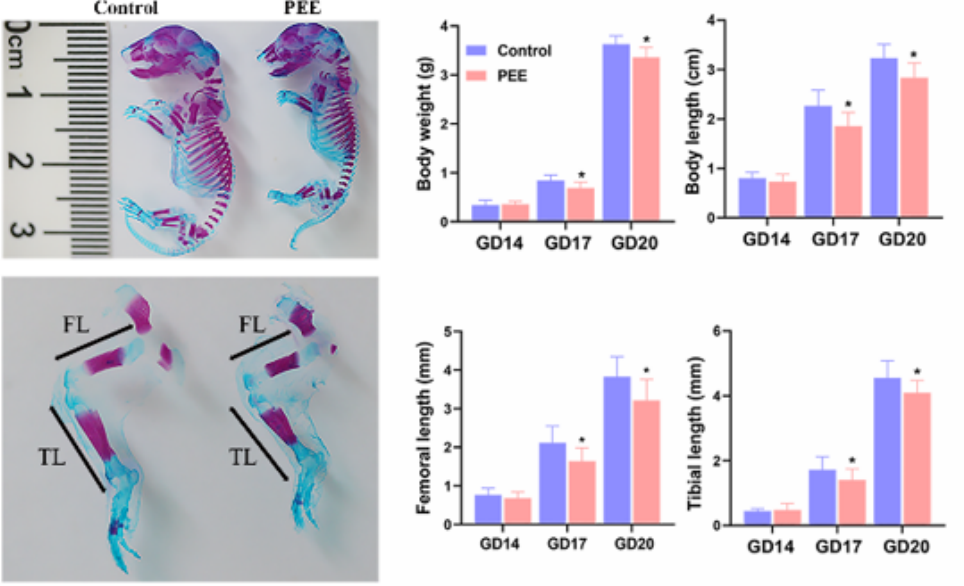

d

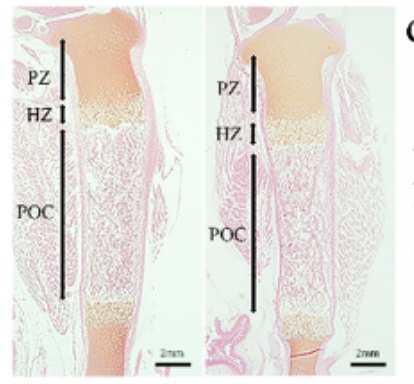

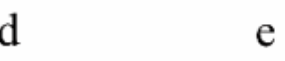

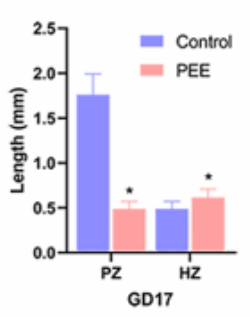

$\mathrm{e}$

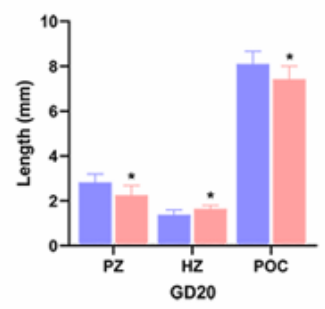

Figure 1

Skeletal and limb development of PEE fetal rats at different times (GD14, GD17, and GD20) during pregnancy. (a) The skeleton shape and limb morphology of PEE fetal rats. (b) Changes in body weight, body length, femur length, and tibia length in PEE fetal rats. (c) Cartilage zones in PEE fetal rats. (d) The widths of the proliferative zone (PZ) and hypertrophic zone (HZ) were measured on GD17. (e) The widths of the $\mathrm{PZ}, \mathrm{HZ}$, and primary ossification center (POC) were measured on GD20. Data are reported as the mean \pm SD. $n=5 .{ }^{*} P<0.05,{ }^{\star} * \mathrm{P}<0.01$, compared with the control group. 
a

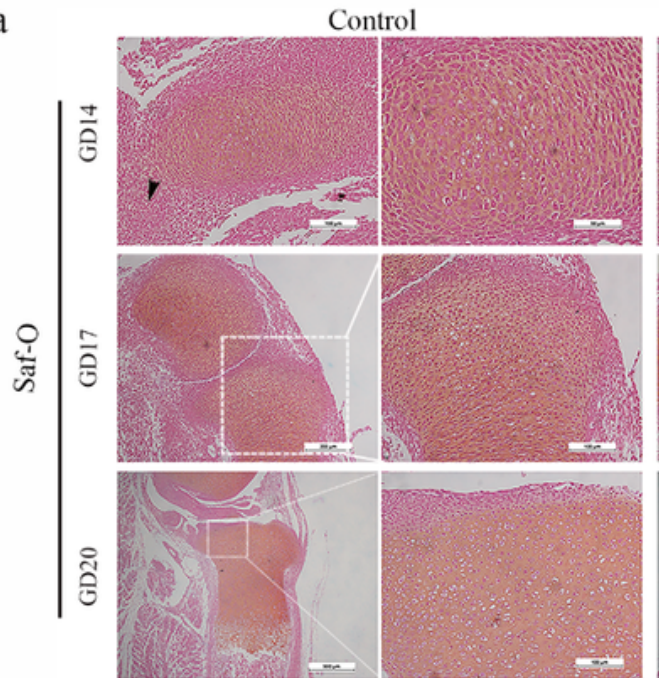

d

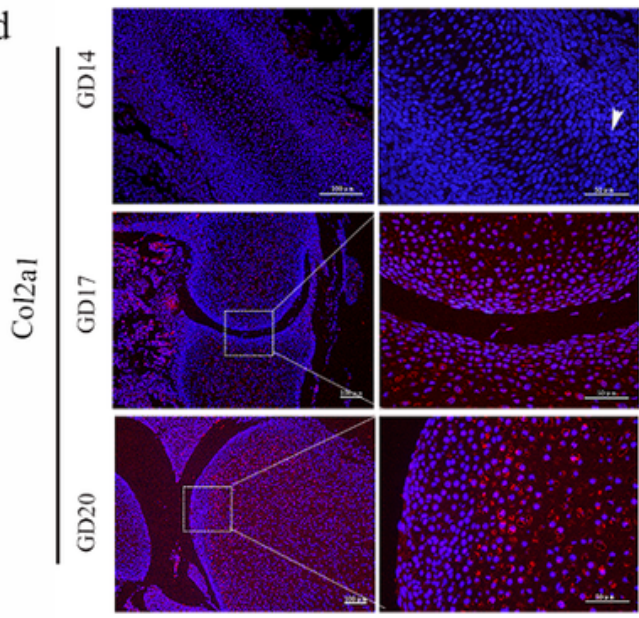

$\mathrm{e}$

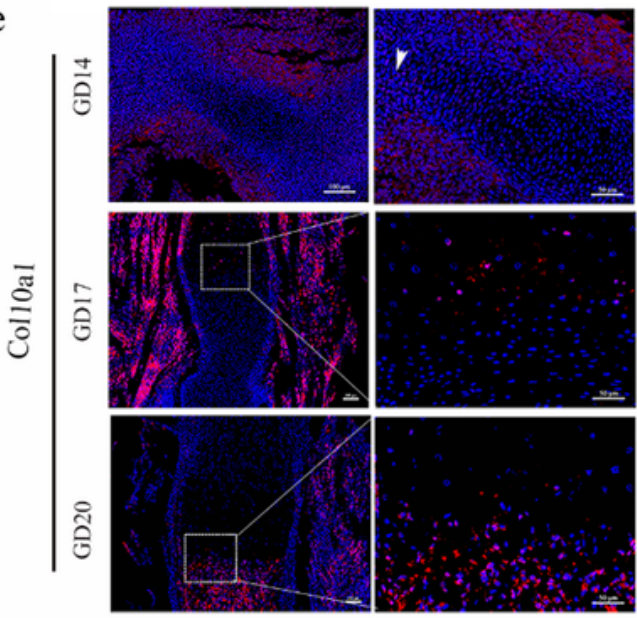

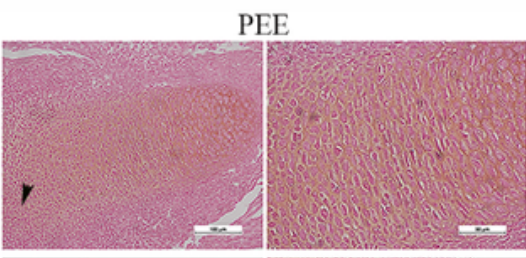
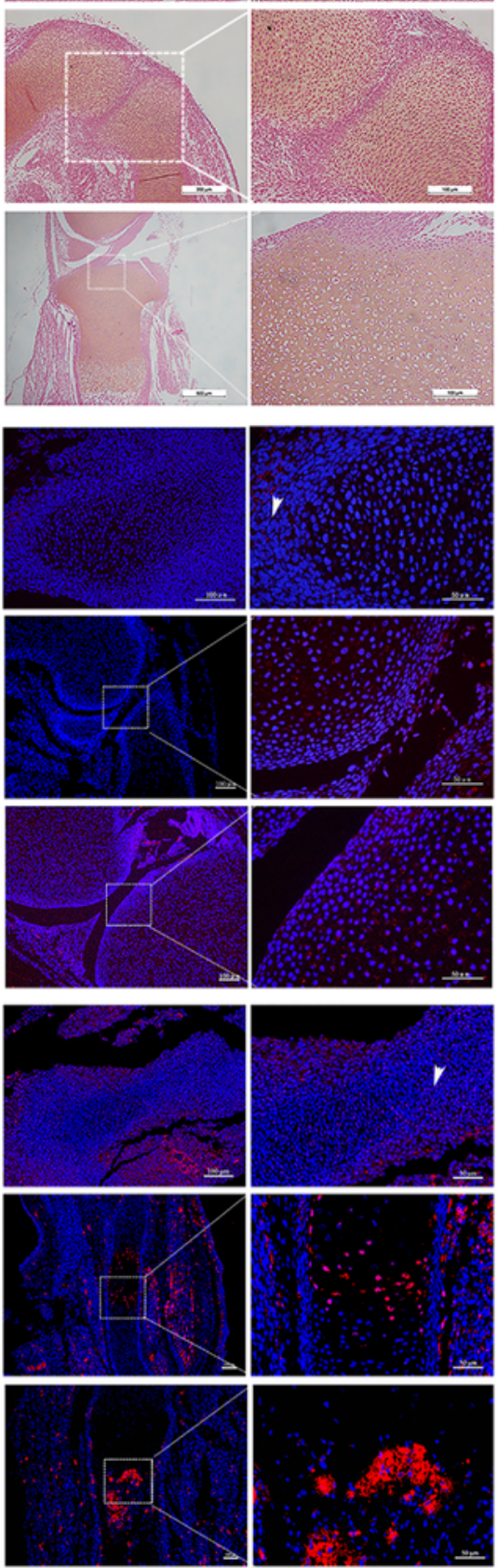

b
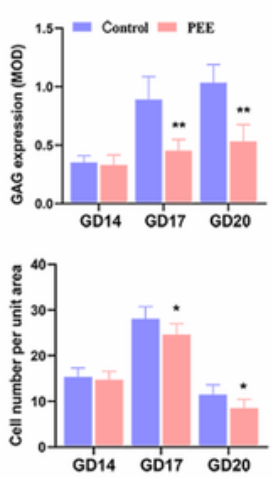

$\mathrm{c}$

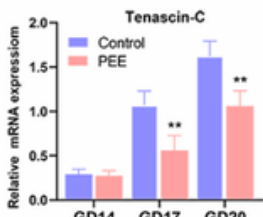

Aggrecan
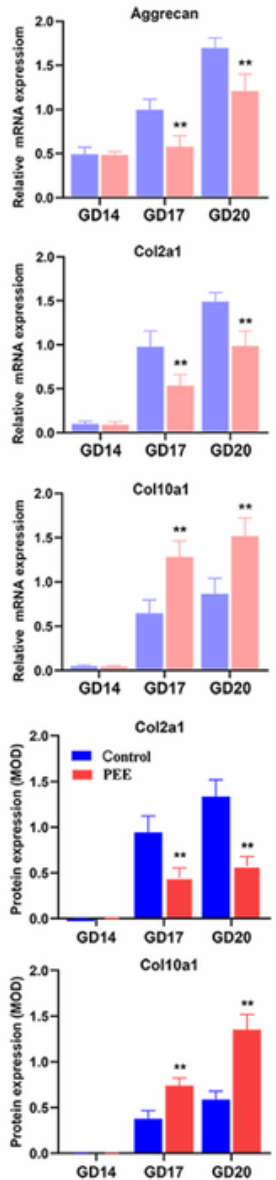

\section{Figure 2}

The development of PEE fetal cartilage at different times (GD14, GD17, and GD20) during pregnancy. (a) Representative Safranin 0 staining of fetal knees at different times during pregnancy. (b) The optical density of glycosaminoglycan (GAG) and cell number per unit area in fetal cartilage. $n=5$. (c) The mRNA expression of tenascin-C, aggrecan, Col2a1, and Col10a1 was analyzed. $n=8$. (d-f) Representative immunofluorescence and mean optical density (MOD) of the chondrocyte marker genes COL2A1 and 
Col10a1 at different times during pregnancy. $n=5$. Data are reported as the mean $\pm S D$. ${ }^{\star} P<0.05, \star \star P<$ 0.01 , compared with the control group.
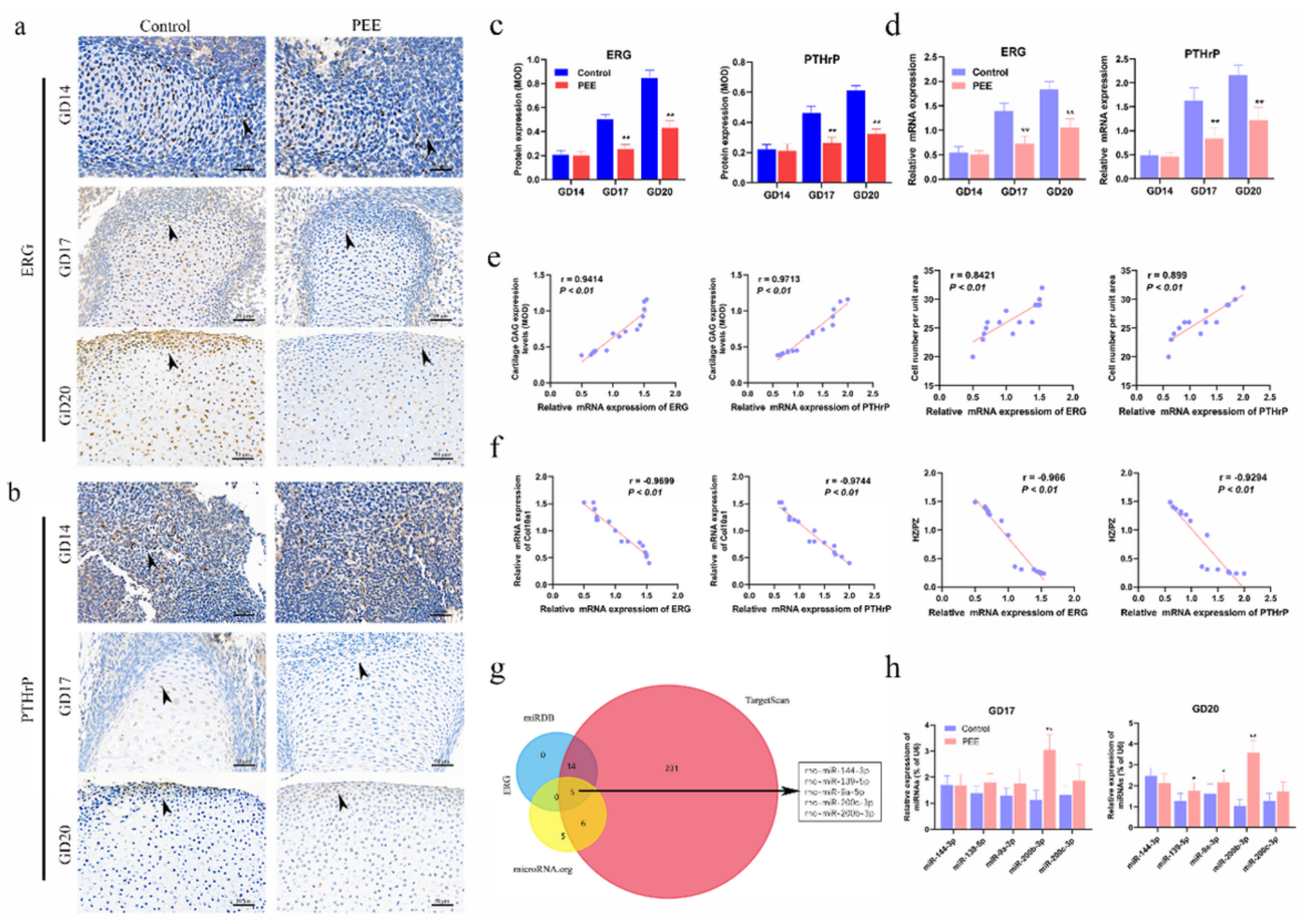

\section{Figure 3}

The expression of ERG, PTHrP, and miRNAs that regulate ERG at different times during pregnancy. (a-c) Representative immunohistochemistry and mean optical density (MOD) of ERG and PTHrP in fetal cartilage. $n=5$. (d) RT-qPCR results of ERG and PTHrP. $n=8$. (e) The mRNA expression of ERG and PTHrP on GD17 was positively correlated with the content of the cartilage matrix (GAG) and the number of cells per unit area. (f) The mRNA expression of ERG and PTHrP was negatively correlated with Col10a1 and $\mathrm{HZ} / \mathrm{PZ}$ on GD17. (g) Predicting the miRNAs that regulate ERG by overlapping the results of the miRDB, TargetScan, and microRNA.org databases. (h) Validation of miRNAs in GD17 and GD20 cartilage tissue samples. $n=8$. Data are reported as the mean $\pm S D$. ${ }^{*} P<0.05$, ${ }^{\star *} P<0.01$, compared with the control group. 


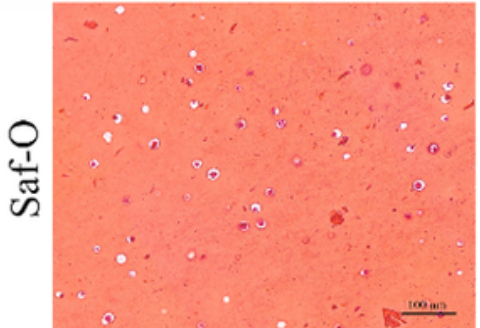

Ethanol (mM) 0

b

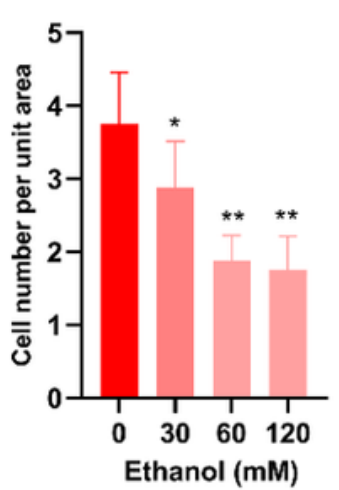

f

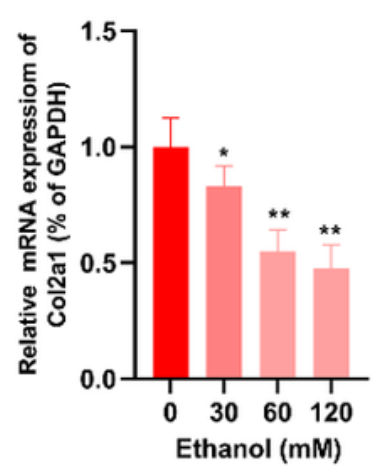

j

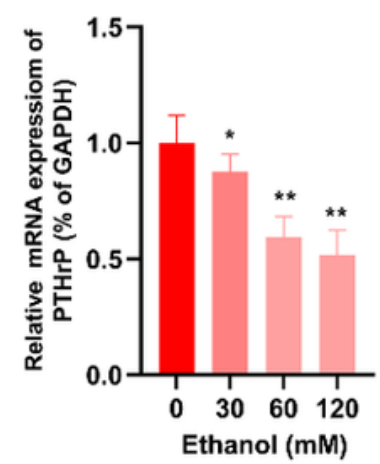

c

g

$\mathrm{k}$

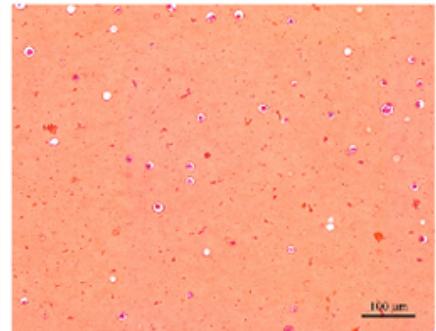

30
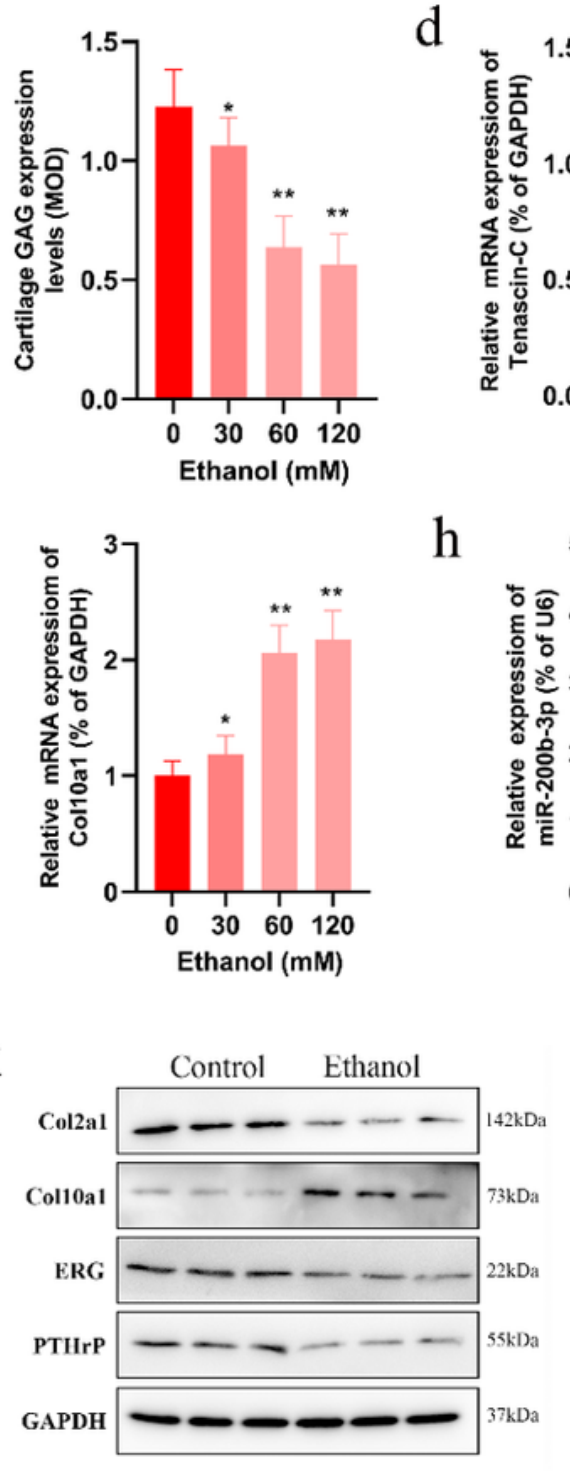

60

d

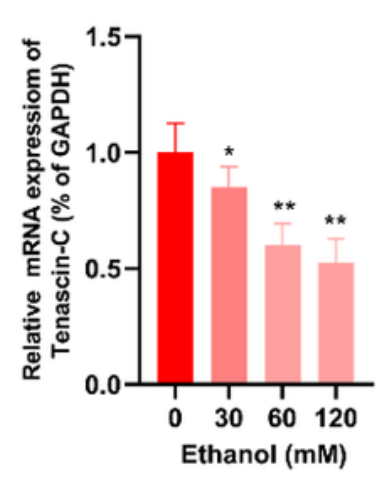

$\mathrm{h}$

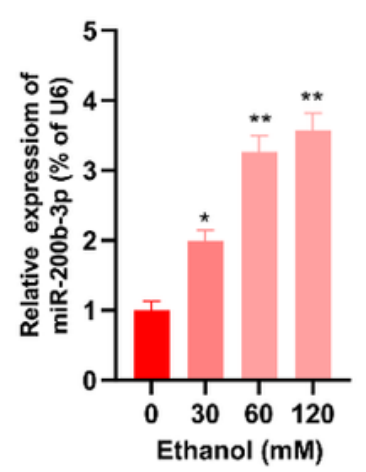

$10 \mathrm{im}$

120

e

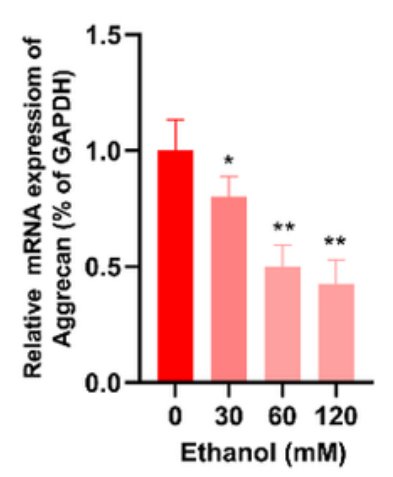

i

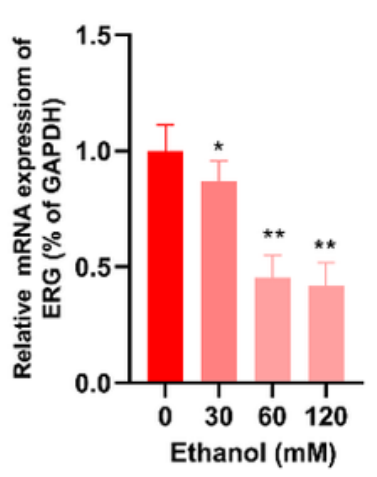

\section{Figure 4}

The effect of ethanol (0-120 mM) on the differentiation of BMSCs to chondrocytes. (a-c) Safranin 0 staining, the number of chondrocytes per unit area, and the GAG content of chondrocytes after the differentiation of BMSCs treated with different concentrations of ethanol. $n=5$. (d-g) The expression of tenascin-C, aggrecan, Col2a1, and Col10a 1 in chondrocytes after the differentiation of BMSCs treated with different concentrations of ethanol. $n=8$. (h-j) The expression of miR-200b-3p, ERG, and PTHrP in 
chondrocytes after the differentiation of BMSCs treated with different concentrations of ethanol. $n=8$. $(k$ I) The protein expression and quantification of Col2a1, Col10a1, ERG, and PTHrP after the differentiation of BMSCs in the control group and the $60 \mathrm{mM}$ ethanol group. $\mathrm{n}=3$. Data are reported as the mean $\pm \mathrm{SD}$. ${ }^{*} \mathrm{P}<0.05,{ }^{* *} \mathrm{P}<0.01$, compared with the control group.
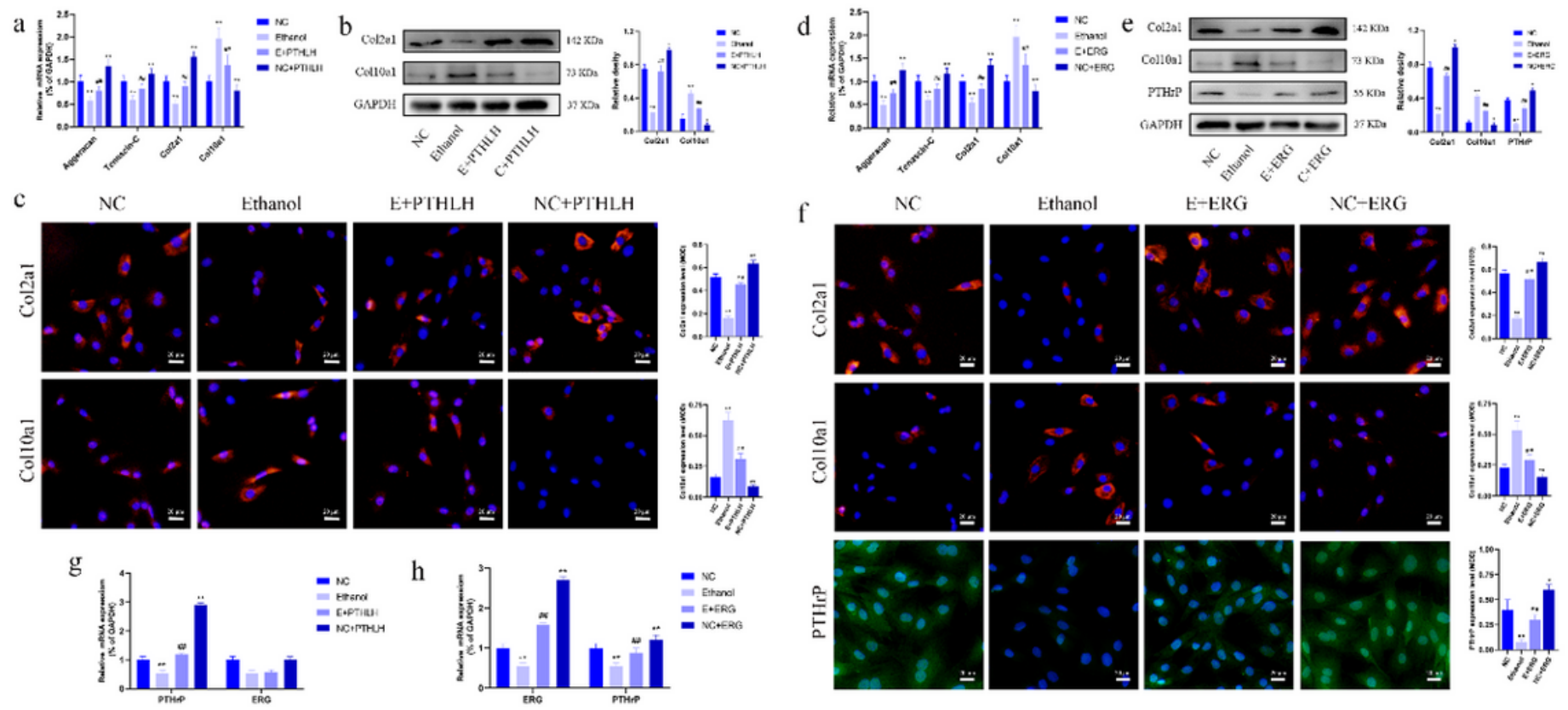

\section{Figure 5}

Overexpression of PTHLH and ERG reversed the ethanol-induced changes in the expression of marker genes in chondrocytes induced. (a-c) Overexpression of PTHLH reversed the ethanol-induced changes in the mRNA expression of tenascin-C, aggrecan, Col2a1, and Col10a1, as shown by RT-qPCR $(n=8)$, western blotting $(n=3)$, and immunofluorescence $(n=3)$. ( $(d-f)$ Overexpression of ERG reversed the ethanol-induced changes in the mRNA expression of tenascin-C, aggrecan, Col2a1, and Col10a1, as shown by RT-qPCR $(n=8)$, western blotting $(n=3)$, and immunofluorescence $(n=3) .(g, h)$ Overexpression of PTHLH had no influence on the ethanol-induced change in the mRNA expression of ERG, while overexpression of ERG reversed the effect of ethanol on the mRNA expression of PTHrP. $n=8$. Data are reported as the mean \pm SD. ${ }^{*} P<0.05,{ }^{\star *} P<0.01$, compared with the NC group; $\# \# P<0.01$, compared with the ethanol group. 
a

635-642 of ERG 3' UTR 5' ... UU--CAGUAUUA ‥

| | | |||

rno-miR-200b-3p $\quad 3^{\prime} \quad$--GUCAUAAU

635-642 of ERG 3' UTR-Mut $\quad 5^{\prime} \quad$--ACUGCGGA $\cdots$

e

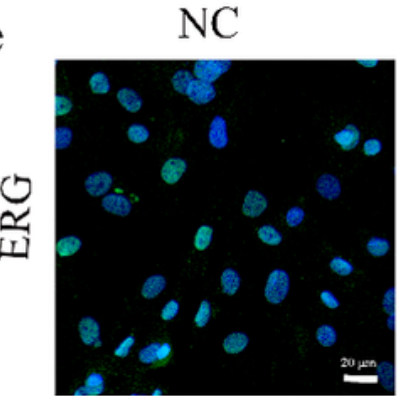

f

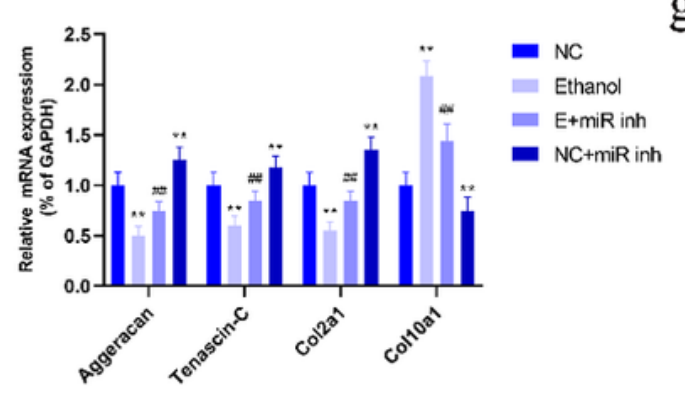

$\mathrm{h}$
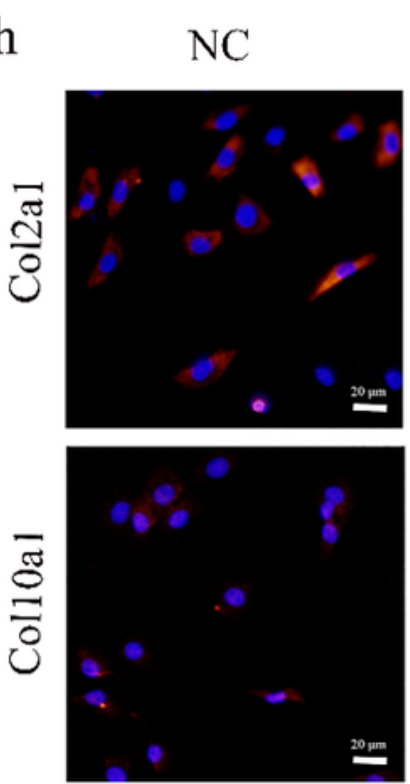

Ethanol

Ethanol b
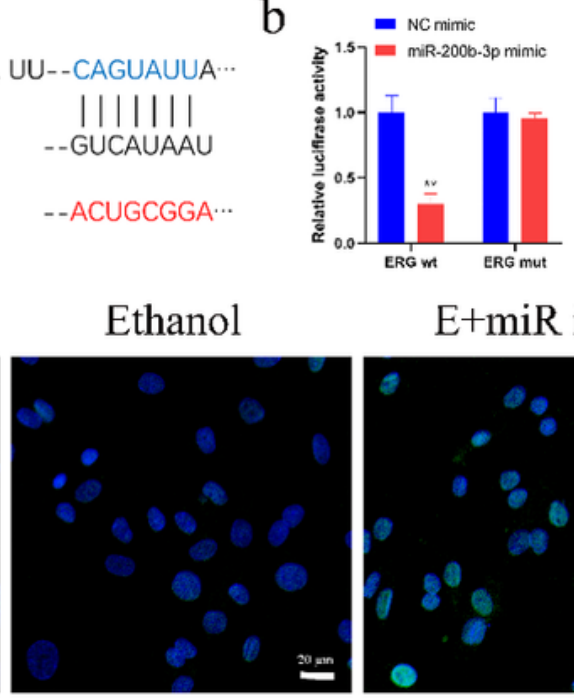

$\mathrm{g}$

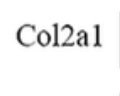

Colloa1

GAPDH

$\mathrm{E}+\mathrm{miR}$ inh

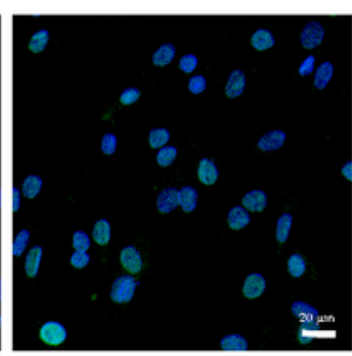

$\mathrm{NC}+\mathrm{miR}$ inh
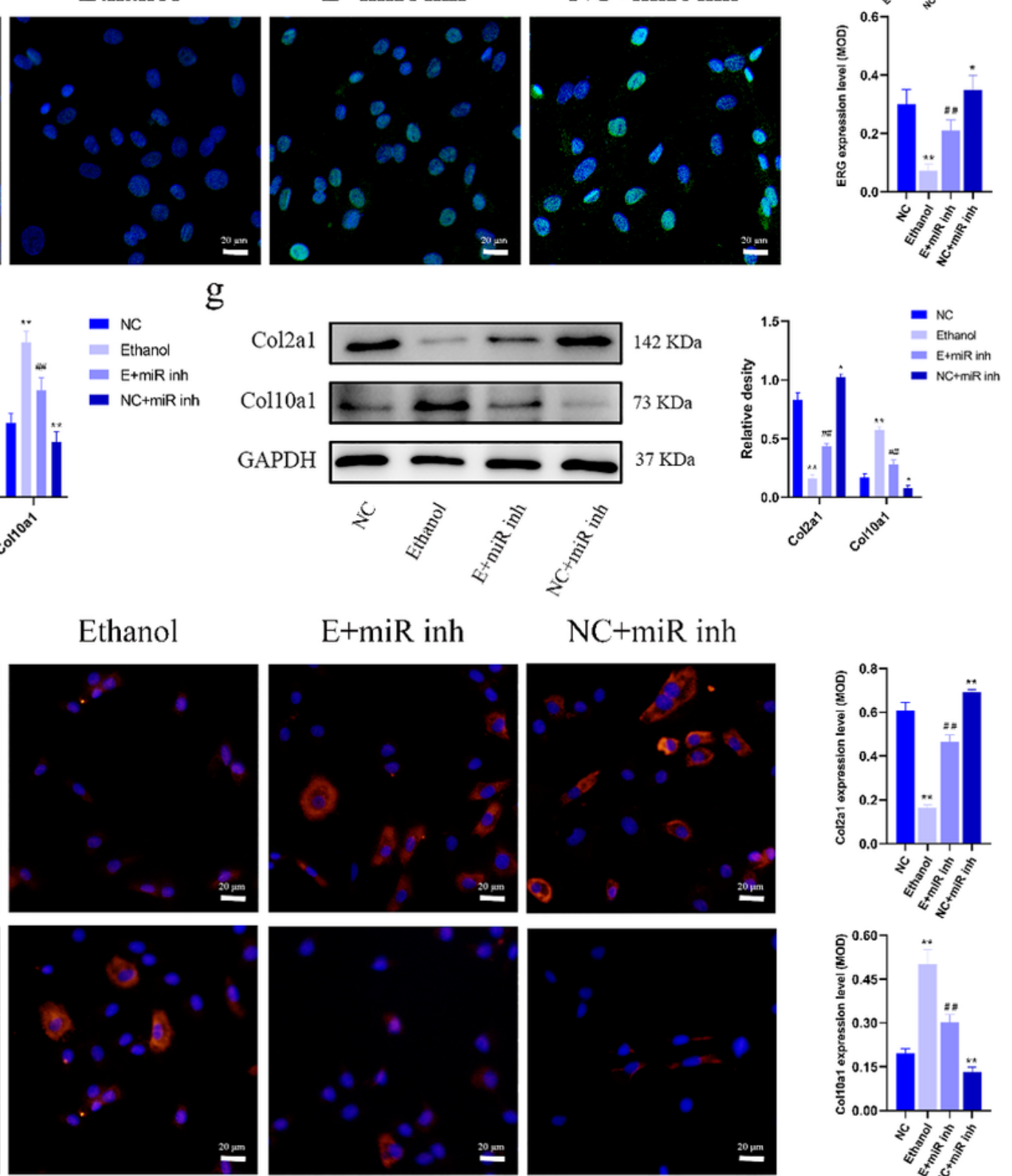

d $\underset{\mathrm{TAPG}}{\mathrm{IRH}}=-$

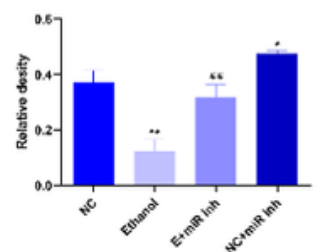

-

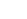
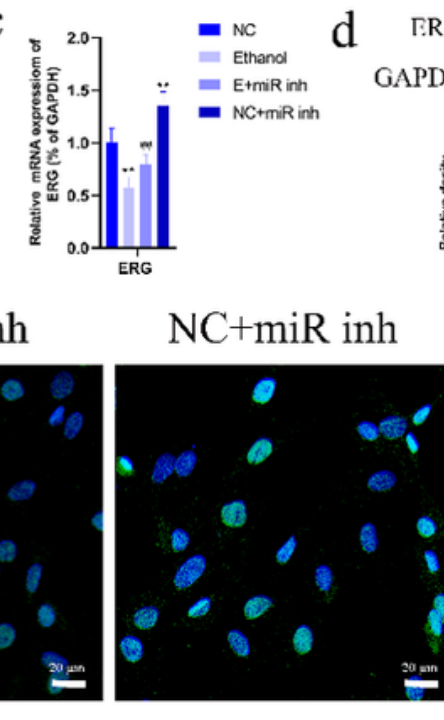

E+miR inh

$\mathrm{NC}+\mathrm{miR}$ inh
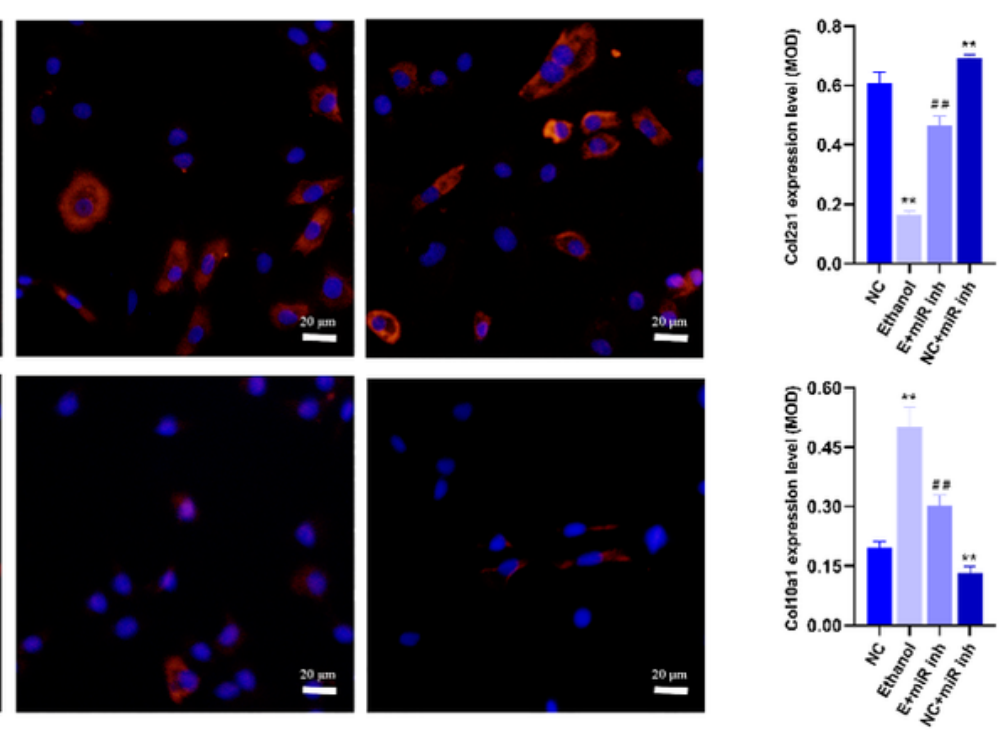

\section{Figure 6}

miR-200b-3p can negatively regulate ERG and chondrocyte differentiation. (a) The binding sequence of miR-200b-3p and ERG. (b) A luciferase reporter assay confirmed that miR-200b-3p could specifically and negatively regulate ERG. $n=3$. (c) RT-qPCR showed that the miR-200b-3p inhibitor could partially reverse the effect of ethanol on the mRNA levels of ERG. n=8. (d-e) Immunofluorescence and western blotting showed that the miR-200b-3p inhibitor could reverse the effect of ethanol on the protein levels of ERG. 
$n=3$. (f) RT-qPCR showed that the miR-200b-3p inhibitor could reverse the effect of ethanol on the mRNA levels of tenascin-C, aggrecan, Col2a1, and Col10a1. (g-h) Immunofluorescence and western blotting showed that the miR-200b-3p inhibitor could reverse the effect of ethanol on the protein levels of tenascin-C, aggrecan, Col2a1, and Col10a1. $n=3$. Data are reported as the mean \pm SD. ${ }^{*} P<0.05,{ }^{*} P<$ 0.01 , compared with the NC group; \#\#P $<0.01$, compared with the ethanol group.

\section{Supplementary Files}

This is a list of supplementary files associated with this preprint. Click to download.

- Supplementarymaterial.docx 